\title{
Analysis of High-Order Finite Elements for Convected Wave Propagation
}

\author{
Hadrien Bériot ${ }^{1 *}$, Gwénaël Gabard ${ }^{2}$ and Emmanuel Perrey-Debain ${ }^{3}$ \\ ${ }^{1}$ LMS International nv Interleuvenlaan 68, Researchpark Z1, 3001 Leuven, Belgium \\ ${ }^{2}$ Institute of Sound and Vibration Research, University Road, Southampton, SO17 BJ, United Kingdom \\ ${ }^{3}$ Laboratoire Roberval, Université de Technologie de Compiègne, 60205 Compiègne Cedex, France
}

\begin{abstract}
SUMMARY
In this paper we examine the performance of high-order finite element methods (FEM) for aeroacoustic propagation, based on the convected Helmholtz equation. A methodology is presented to measure the dispersion and amplitude errors of the $p$-FEM, including non-interpolating shape functions, such as 'bubble' shape functions. A series of simple test cases are also presented to support the results of the dispersion analysis. The main conclusion is that the properties of $p$-FEM that make its strength for standard acoustics (e.g. exponential $p$-convergence, low dispersion error) remain present for flow acoustics as well. However, the flow has a noticeable effect on the accuracy of the numerical solution, even when the change in wavelength due to the mean flow is accounted for, and an approximation of the dispersion error is proposed to describe the influence of the mean flow. Also discussed is the so-called aliasing effect which can reduce the accuracy of the solution in the case of downstream propagation. This can be avoided by an appropriate choice of mesh resolution. Copyright (c) 0000 John Wiley \& Sons, Ltd.
\end{abstract}

Received ...

KEY WORDS: Finite element method; dispersion analysis; high-order elements; $p$-FEM; convected propagation

\section{INTRODUCTION}

When applied to the Helmholtz equation, the conventional finite element method (FEM) is known to suffer from numerical dispersion and the so-called pollution error [1]. Since the dispersion error increases for increasing frequency, the mesh resolution required to obtain a reasonable accuracy also increases with frequency. As a consequence, the use of the standard FEM is restricted in practice to low frequencies. To circumvent this limitation, innovative numerical methods have been proposed over the past two decades, amongst which are the partition of unity method [2], the discontinuous enrichment method [3], and the ultraweak variational method [4]. These methods have in common their reliance on fundamental solutions of the governing equation for the construction of their approximation bases. Although these methods are very promising and have proven their efficiency in addressing the pollution problem, they still suffer from a number of drawbacks. Firstly, they rely on a frequency dependent approximation space which leads to computationally intensive assembly procedures due to the evaluation of oscillatory integrals, which has to be repeated for each frequency. Secondly, they often yield ill-conditioned matrices which prevents the use of efficient iterative solvers. Finally, they are inherently less adapted for problems with inhomogeneous media, likely

${ }^{*}$ Correspondence to: LMS International nv Interleuvenlaan 68, Researchpark Z1, 3001 Leuven, Belgium. E-mail: hadrien.beriot@1msintl.com 
to be encountered in many applications. In particular, their generalization to the modeling of sound propagation in the presence of a non-uniform background flow remains an area of active research $[5,6,7]$. For these reasons, high-order FEM or $p$-FEM methods, which rely on polynomial bases, still represent an efficient and viable alternative for many wave propagation applications $[8,9]$.

Several studies can be found examining the performance of the $p$-FEM for solving the Helmholtz equation. A common approach consists in solving the dispersion relation of the discretized wave operator. These dispersion analyses are typically performed on periodic mesh stencils, and so the boundary conditions are not included. The dispersion error can then be obtained either numerically by searching for the roots of the finite element equations, or analytically by finding a closed-form expression of the discrete dispersion relation. While analytical derivations are typically obtained in one dimension, and extended to the two-dimensional (quadrangle) and three-dimensional (hexahedron) cases through tensor products, numerical analysis allows one to address directly a larger variety of mesh topologies. Thompson and Pinsky [10] were among the first to focus on the dispersion properties of higher-order FEM for the Helmholtz equation. They perform a onedimensional numerical dispersion analysis for different polynomial bases up to the interpolation order $P=5$. The results indicate that the dispersion relation for a $P$-th order scheme is accurate to order $2 P$ when $k h$ tends to zero, where $k$ is the wavenumber and $h$ is the element size. Mulder [11] also performed a numerical dispersion analysis of high-order methods for the one-dimensional Helmholtz equation, with special emphasis on the so-called 'spectral techniques', based on highorder Lagrange polynomial interpolation. He evaluated the effects of different nodal sampling (e.g. regular or Chebyshev-based) on the dispersion error for the order $P=1$ to 6 .

An important aspect of reference [11] was to demonstrate that for high-order methods one needs to consider not only the dispersion error but also the amplitude error to obtain a complete assessment of the dispersion properties. In a series of papers, Ihlenburg and Babuška $[12,13]$ propose a mathematical analysis of the dispersion properties of general Galerkin finite elements for Helmholtz equation. They prove that the pollution effect is closely linked to the dispersion error and demonstrate its low frequency asymptotic behavior (e.g. $k h \ll 1$ ) in $2 P$. Later on, Deraemaeker et al. [14] performed a numerical analysis of various finite element schemes for the Helmholtz equation in two and three dimensions. In particular, they present results for the integrated Legendre quadrangle and hexahedron elements up to the order 4. Their analysis demonstrated that for a given number of unknowns, the $p$-FEM drastically reduces the pollution error. Ainsworth [15] generalized the work of Ilhenburg and Babuška by deriving analytical expressions at all orders for different discretization rates, including the asymptotic low frequency regime $k h \ll 1$ where the $p$-convergence is exponential, as well as three different regimes at high frequency.

A prominent feature of the $p$-FEM is the presence of significant peaks in both the dispersion and amplitude errors for specific mesh resolutions. They are related to the bubble shape functions, which are internal degrees of freedom in each element that can be eliminated using static condensation. As indicated in $[12,13]$, the peaks of error correspond to the eigenvalues of the condensation problem. Using an analogy with crystal lattices, Thompson and Pinsky [10] describe this phenomenon as band gaps in the dispersion relation while Cottrell et al. [16] refer to the existence of so-called optical branches in the dispersion relation (see also [17]). Mulder [11] interprets this feature as an aliasing effect between wavenumber components supported by the discrete wave operator.

In parallel to dispersion analyses, performance evaluations on numerical benchmarks also demonstrate the significant benefits of the $p$-FEM over conventional low-order FEM for the Helmholtz equation. In [18], several polynomial bases commonly used in spectral and FE methods (Lagrange, integrated Legendre and Bernstein shape functions) are compared. High-order polynomial approximations are proved to effectively control the pollution and to lead to more efficient simulations. Bernstein polynomials are also shown to be superior when resorting to Krylov subspace iterative solutions. Vos et al. [19] investigate various implementation strategies of the integrated Legendre polynomials for different operators and compare their computational efficiency. Computational tests are performed on different Helmholtz problems with a large number of $h$ and $p$-refinement combinations. Results indicate that the optimal performance for a given error tolerance is typically obtained with an order 5 or 6 . The advantage of benchmark analysis in comparison with 
the dispersion analysis lies in the fact that they incorporate all sources of errors likely to appear in real-life computations, such as boundary conditions and local mesh irregularities. In addition, the numerical efficiency can be assessed by comparing run-times, memory consumption, etc. However, the conclusions of these engineering analyses remain dependent on the choice of the benchmark parameters (mesh resolution, frequency, boundary conditions), the choice of the implementation strategy and the choice of the computational resources and, as such, results are less general than those provided by the dispersion analysis.

In the present paper, we are interested in predicting sound propagation in moving media, which is of particular importance for various industrial applications such as aircraft noise. Compared to the standard Helmholtz problem, the number of dispersion analyses of FE methods for aeroacoustics applications is quite limited. To the authors' knowledge, Gabard et al. [20, 21] were the first to examine the dispersion properties of the finite elements for the convected wave equation. The amplitude error was also taken into account in the analysis, as in [11]. They examined the sensitivity of the numerical model to the wave and/or the flow directions, and highlight some important aliasing effects in the case of downstream propagation. However, their analysis was restricted to conventional linear and quadratic finite element shape functions. Here, we intend to extend the study to higherorder finite elements, by analyzing the performance of integrated Legendre $p$-FEM shape functions for the convected wave equation.

This paper is organized as follows: in Section 2 we begin by introducing the convected Helmholtz equation, its variational formulation and the associated boundary conditions. The definition and properties of the integrated Legendre shape functions are also presented. In Section 3, a onedimensional dispersion analysis is performed for the $p$-FEM elements up to the order 10. A simple one-dimensional benchmark boundary value problem is also solved to relate the findings of the dispersion analysis to the numerical error observed in practice. A two-dimensional dispersion analysis is then presented in Section 4 to study the anisotropy induced by the mesh topology.

\section{HIGH-ORDER ELEMENTS FOR THE CONVECTED HELMHOLTZ EQUATION}

\subsection{Governing equations and variational formulation}

We consider acoustic perturbations of small amplitude propagating on a mean flow. For the purpose of the present work we can consider a uniform flow with mean velocity $\mathbf{v}_{0}$ and sound speed $c_{0}$. For the sake of conciseness, we will work with non-dimensional variables by setting the sound speed $c_{0}$ and the mean flow density $\rho_{0}$ to unity. The perturbations are modelled in the frequency domain, assuming an implicit time dependence given by $\mathrm{e}^{-\mathrm{i} \omega t}$. This problem can be described by the convected Helmholtz equation written for the acoustic pressure $p$ :

$$
\frac{\mathrm{d}_{0}^{2} p}{\mathrm{~d} t^{2}}-\nabla^{2} p=0
$$

where $\mathrm{d}_{0} / \mathrm{d} t=-\mathrm{i} \omega+\left(\mathbf{v}_{0} \cdot \nabla\right)$ is the material derivative in the mean flow. This equation can be expressed as a weak variational statement over a domain $\Omega$ with boundary $\Gamma$ (see [22] for more details):

$$
\int_{\Omega}-\overline{\frac{\mathrm{d}_{0} w}{\mathrm{~d} t}} \frac{\mathrm{d}_{0} p}{\mathrm{~d} t}+\nabla \bar{w} \cdot \nabla p \mathrm{~d} \Omega+\int_{\Gamma} \bar{w}\left[\left(\mathbf{v}_{0} \cdot \mathbf{n}\right) \frac{\mathrm{d}_{0} p}{\mathrm{~d} t}-\frac{\partial p}{\partial \nu}\right] \mathrm{d} \Gamma=0, \quad \forall w,
$$

where $w$ is the test function, the overbar denotes the complex conjugate and $\mathbf{n}$ is the outward unit normal on $\Gamma$ and $\partial p / \partial \nu=\nabla p \cdot \mathbf{n}$. For a hard wall, we have $\mathbf{v}_{0} \cdot \mathbf{n}=0$ and $\partial p / \partial \nu=0$ and the boundary integral vanishes. We shall also use a Robin boundary condition (generalized to include the mean flow effect):

$$
\frac{\partial p}{\partial \nu}-\frac{\mathrm{i} \omega}{1+\mathbf{v}_{0} \cdot \mathbf{n}} p=g
$$



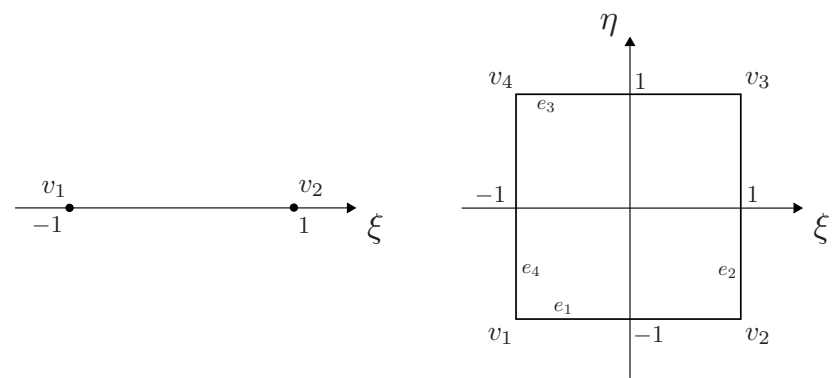

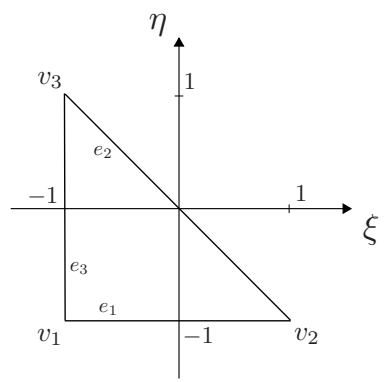

Figure 1. Reference elements $E^{L}(\xi)$ (left), $E^{Q}(\xi, \eta)$ (center) and $E^{T}(\xi, \eta)$ (right).

where $g$ is a given source term. The corresponding boundary integral in the variational formulation then reads:

$$
\int_{\Gamma} \bar{w}\left\{\left[\left(\mathbf{v}_{0} \cdot \mathbf{n}\right)^{2}-1\right] g-\mathrm{i} \omega p\right\} \mathrm{d} \Gamma,
$$

where we have assumed that the mean flow is normal to the boundary.

With no flow, equations (1) and (2) reduce to the standard formulation for Helmholtz problems. With flow the main difference is the term $\mathbf{v}_{0} \cdot \nabla$ introduced by the material derivative. This adds an element of convection in addition to the standard Helmholtz equation.

\subsection{High-order discretization}

The computational domain $\Omega$ is partitioned into a set of non-overlapping elements. In this study, we consider line, triangle and quadrilateral elements, denoted by $E^{L}, E^{Q}$ and $E^{T}$ respectively, and defined in Figure 1.

We use the classical $H^{1}$-conforming hierarchic $p$-FEM shape functions (see Šolín et al. [23] for details). The hierarchic shape functions are defined such that the finite element basis of degree $P+1$ is obtained as a correction to the basis of degree $P$. For one-dimensional analysis the hierarchic shape functions of order $P=1$ defined on the reference element $E^{L}=[-1 ; 1]$ are the standard linear shape functions:

$$
l_{0}(\xi)=\frac{1-\xi}{2}, \quad l_{1}(\xi)=\frac{\xi+1}{2} .
$$

The functional basis for an element of order $P$ is then formed by appending $P-1$ shape functions of the form

$$
l_{q}(\xi)=\sqrt{\frac{2 q-1}{2}} \int_{-1}^{\xi} L_{q-1}(s) \mathrm{d} s, \quad \text { with } q=2, \ldots, P,
$$

where $L_{q}(\xi)$ is the Legendre polynomial of order $q$. These integrated Legendre polynomials, also called Lobatto functions, verify the following orthogonality property:

$$
\int_{-1}^{1} \frac{\partial l_{i}}{\partial \xi} \frac{\partial l_{j}}{\partial \xi} \mathrm{d} \xi=\delta_{i j}, \quad \text { with } i, j=0,1, \ldots, P, \quad \text { and } i \text { or } j \geqslant 2 .
$$

This property has the benefit of minimizing the conditioning of the stiffness matrix for Helmholtz problems [24]. The numerical approximation $\tilde{p}_{n}$ over an element $n$, is constructed as the sum of two contributions:

$$
\tilde{p}_{n}(\xi)=\tilde{p}_{n}^{v}(\xi)+\tilde{p}_{n}^{b}(\xi)
$$

with

$$
\tilde{p}_{n}^{v}(\xi)=l_{0}(\xi) \alpha_{n}^{v}+l_{1}(\xi) \alpha_{n+1}^{v}, \quad \text { and } \quad \tilde{p}_{n}^{b}(\xi)=\sum_{q=2}^{P} \alpha_{q, n}^{b} l_{q}(\xi),
$$

where $\tilde{p}_{n}^{v}$ is the standard linear nodal interpolation (with degrees of freedom $\alpha_{n}^{v}$ ) while $\tilde{p}_{n}^{b}$ represents the hierarchic bubble enrichment functions added within each element $\left(\alpha_{q, n}^{b}\right)$ denotes the bubble degrees of freedom of order $q$ in element $n$ ). 
The shape functions on the quadrilateral reference element $E^{Q}$ are easily obtained by taking a tensor product of the one-dimensional shape functions. On the triangle reference element $E^{T}$, the construction of the shape functions is less trivial, as they are constructed through a specific generalized mapping which matches the corresponding quadrilateral shapes [19]. The triangle reference element defined in Figure 1 is first equipped with affine coordinates

$$
\lambda_{1}(\xi, \eta)=(\eta+1) / 2, \quad \lambda_{2}(\xi, \eta)=-(\eta+\xi) / 2, \quad \lambda_{3}(\xi, \eta)=(\xi+1) / 2 .
$$

The numerical approximation $\tilde{p}$ over a triangle element is constructed as the sum of the nodal, edge and bubble functions contributions as follows

$$
\tilde{p}(\xi, \eta)=\tilde{p}^{v}(\xi, \eta)+\tilde{p}^{e}(\xi, \eta)+\tilde{p}^{b}(\xi, \eta) .
$$

The nodal contribution reads

$$
\tilde{p}^{v}(\xi, \eta)=\sum_{r=1}^{3} \varphi^{v_{r}}(\xi, \eta) \alpha^{v_{r}},
$$

where $\varphi^{v_{r}}$ denote the conventional linear shape functions

$$
\varphi^{v_{1}}=\lambda_{2}(\xi, \eta), \quad \varphi^{v_{2}}=\lambda_{3}(\xi, \eta), \quad \varphi^{v_{3}}=\lambda_{1}(\xi, \eta),
$$

and $\alpha^{v_{r}}$ the corresponding nodal degrees of freedom. The edge contribution reads

$$
\tilde{p}^{e}(\xi, \eta)=\sum_{q=2}^{P} \sum_{r=1}^{3} \varphi_{q}^{e_{r}}(\xi, \eta) \alpha_{q}^{e_{r}},
$$

where $\alpha_{q}^{e_{r}}$ denotes the degree of freedom of order $q$ of the $r^{\text {th }}$ edge and $\varphi^{e_{r}}$ the corresponding edge shape functions

$$
\varphi_{q}^{e_{1}}=\lambda_{2} \lambda_{3} \Psi_{q-2}\left(\lambda_{3}-\lambda_{2}\right), \quad \varphi_{q}^{e_{2}}=\lambda_{3} \lambda_{1} \Psi_{q-2}\left(\lambda_{1}-\lambda_{3}\right), \quad \varphi_{q}^{e_{3}}=\lambda_{1} \lambda_{2} \Psi_{q-2}\left(\lambda_{2}-\lambda_{1}\right),
$$

with $2 \leq q \leq P$, where we have introduced the following kernel function

$$
\Psi_{q}(x)=\frac{l_{q+2}(x)}{l_{0}(x) l_{1}(x)} .
$$

The edge shape functions are the traces of the integrated Legendre functions on one of the edges and vanish elsewhere on the element boundary. Finally, the bubble functions read

$$
\tilde{p}^{b}(\xi, \eta)=\sum_{q_{1}=1}^{P} \sum_{q_{2}=1}^{P-1-q_{1}} \varphi_{q_{1}, q_{2}}^{b}(\xi, \eta) \alpha_{q_{1}, q_{2}}^{b},
$$

where $\varphi_{q_{1}, q_{2}}^{b}$ denotes the following bubble shape functions [23]

$$
\varphi_{q_{1}, q_{2}}^{b}=\lambda_{1} \lambda_{2} \lambda_{3} \Psi_{q_{1}-1}\left(\lambda_{3}-\lambda_{2}\right) \Psi_{q_{2}-1}\left(\lambda_{2}-\lambda_{1}\right), \quad \text { with } 1 \leq q_{1}, q_{2} \text { and } q_{1}+q_{2} \leq P-1,
$$

and $\alpha_{q_{1}, q_{2}}^{b}$ are the corresponding bubble degrees of freedom. To evaluate the elementary integrals described in (2), one has to numerically integrate the products of higher-order shape functions and their derivatives. High-order numerical quadrature is used for this purpose with an order of accuracy corresponding to the highest polynomial order that appears in the integrand. For the line and the quadrangle elements, standard Gauss-Legendre quadrature is used. For the triangle elements, we use the Dunavant economical quadrature rules [25]. Due to their hierarchic structure, the integrated Legendre basis functions are very flexible and neighbouring elements may have different interpolation orders (localized $p$-refinements). In fact, it has been shown that some interior shape functions can be rather ineffective and may not yield a large improvement in accuracy. For instance, Deraemaeker et al. [14] show that the optimal interpolation scheme is obtained by maintaining a $P-1$-th order for the bubble functions, while considering edge shape functions of order $P$. However, for the sake of simplicity, only complete polynomial spaces of order $P$, where $P$ is uniform over the entire mesh will be considered in this study. 


\section{ONE-DIMENSIONAL ANALYSIS}

\subsection{Dispersion relation of the numerical model}

We consider an infinite, periodic mesh with nodes $x_{n}$ evenly spaced with distance $h$ as depicted in Figure 2.

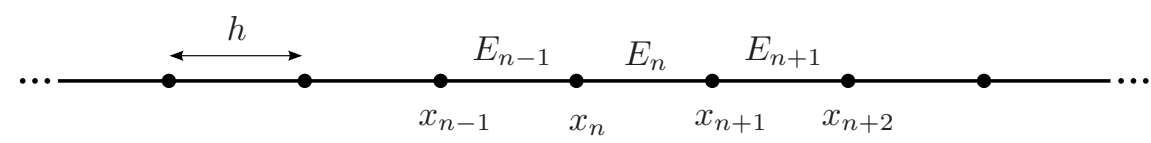

Figure 2. Mesh used for the one-dimensional dispersion analysis.

Each element $E_{n}=\left[x_{n}, x_{n+1}\right]$ of the infinite mesh is mapped onto the usual reference element $E^{L}=[-1,1]$. In the absence of boundary terms, the discretization of the weak form (2) leads to an algebraic system of the form

$$
\mathrm{R}_{\infty} \phi_{\infty}=\mathbf{0}
$$

where $\mathrm{R}_{\infty}$ is a coefficient matrix and $\phi_{\infty}$ the column vector containing the degrees of freedom. The unknowns are ordered as follows:

$$
\phi_{\infty}=\left(\ldots, \phi_{n-1}, \phi_{n}, \phi_{n+1}, \ldots\right)^{T},
$$

where $\phi_{n}$ is the line vector

$$
\phi_{n}=\left(\alpha_{n}^{v}, \alpha_{2, n}^{b}, \alpha_{3, n}^{b}, \ldots, \alpha_{P, n}^{b}\right)
$$

containing the degree of freedom associated to the node $n$ and the internal degrees of freedom of the element $E_{n}$. Since the mesh and the interpolation are periodic, the equations satisfied by the degrees of freedom $\phi_{n}$ are the same as for $\phi_{n+1}$ and the matrix $\mathrm{R}_{\infty}$ can be constructed from a finite number $N$ of equations. If we consider the equation associated with the $q^{\text {th }}$ component of the vector $\phi_{n}$, we can write this equation as follows:

$$
\sum_{m=1}^{N} \sum_{n} A_{n}^{(q, m)} \phi_{n}^{(m)}=0, \quad \text { for } q=1,2, \ldots, N,
$$

where $\phi_{n}^{(m)}$ denotes the $m^{\text {th }}$ component of $\phi_{n}$ and $A_{n}^{(q, m)}$ its contribution in the equation for the component $q$. This contribution is non-zero if and only if the corresponding shape functions overlap. To find the dispersion relation of the numerical model, we seek harmonic solutions of the form:

$$
p(x)=\mathrm{e}^{\mathrm{i} k x} .
$$

By definition of the nodal degrees of freedom, we have: $\tilde{p}_{n}(\xi=-1)=\alpha_{n}^{v}$ and $\tilde{p}_{n}(\xi=1)=\alpha_{n+1}^{v}$, and it follows that

$$
\alpha_{n}^{v}=\mathrm{e}^{\mathrm{i} k x_{n}}=\tau^{n},
$$

where we have introduced the parameter $\tau=\mathrm{e}^{\mathrm{i} k h}$. Expression (23) easily generalizes to any nodal shape functions (e.g. based on Lagrange shape functions) irrespectively of the interpolation order or the topology of the periodic, infinite mesh. Any nodal degree of freedom $\phi_{n}^{(1)}$ can thus be written as a harmonic wave with complex amplitude $\phi_{0}^{(1)}$ :

$$
\phi_{n}^{(1)}=\phi_{0}^{(1)} \tau^{n},
$$

where the complex amplitude $\phi_{0}^{(1)}$ does not depend on the element number $n$. If the approximation basis only contains nodal functions, the numerical dispersion relation is then readily obtained [14].

In the context of hierarchic finite element interpolation, generalizing (24) to show that all unknowns can be reduced to the form (24) is not trivial, given that the amplitude $\alpha_{q, n}^{b}$ of the bubble 
functions are not directly related to nodal values of the solution (22). We follow here the idea of projection-based interpolation presented by Šolín et al. [23].

The amplitudes of the bubble functions are chosen so as to minimize the residue $r=p-\tilde{p}_{n}=$ $p-\tilde{p}_{n}^{v}-\tilde{p}_{n}^{b}$ over each $E_{n}$ element. Here $r$ is minimized in the semi-norm $H^{1}\left(E_{n}\right)$ (see [23] for details). This yields

$$
\int_{-1}^{1} \frac{\partial r}{\partial \xi} \frac{\partial l_{m}}{\partial \xi} \mathrm{d} \xi=0, \quad m=2,3, \ldots, P
$$

which indicates that the residue is orthogonal to the Lobatto functions. Using the orthogonality property (6) of the Lobatto functions, one finds

$$
\alpha_{q, n}^{b}=\mathrm{i} k \frac{h}{2} \int_{-1}^{1} \frac{\partial l_{q}}{\partial \xi} \mathrm{e}^{\mathrm{i} k \frac{h}{2}(\xi+1)} \mathrm{d} \xi\left(\mathrm{e}^{\mathrm{i} k h}\right)^{n}, \quad q=2,3, \ldots, P .
$$

This expression highlights the fact that the contributions of the bubble shape functions are also of the form (24), and any degree of freedom of the vector $\phi_{n}$ satisfies

$$
\phi_{n}^{(m)}=\phi_{0}^{(m)} \tau^{n}, \quad m=1, \ldots, N .
$$

Substituting (27) into (21), one can derive a linear system of size $N=P$ of the form:

$$
\mathrm{R}(\omega, k) \phi_{0}=\mathbf{0}
$$

with $\phi_{0}=\left(\phi_{0}^{(1)}, \phi_{0}^{(2)}, \ldots, \phi_{0}^{(N)}\right)^{T}$. This system represents the dispersion relation of the numerical model. For a fixed angular frequency $\omega$, the 'discrete wavenumbers' $\tilde{k}$ to this dispersion relation can be sought by solving $\operatorname{det}(\mathrm{R}(\omega, k))=0$. Except for linear or very low-order $P$, the $k$-dependency (or equivalently $\tau$-dependency) of the system matrix $\mathrm{R}$ is not trivial and non-linear optimization algorithms such as the Gauss-Newton scheme are used to find the solutions of the dispersion relation.

\subsection{Dispersion and amplitude errors}

For a fixed value of the angular frequency $\omega$, when combining the equations (22) and (1) one finds that the convected Helmholtz equation supports two exact solutions with distinct wavenumbers:

$$
k^{ \pm}=\frac{\omega}{M \pm 1}
$$

where $M=v_{0} / c_{0}$ is the mean flow Mach number. These correspond to the wave propagating in the positive $\left(k^{+}\right)$or negative $\left(k^{-}\right)$direction. The dispersion error is then defined as follows:

$$
E_{d}=\left|\frac{k-\tilde{k}}{k}\right| \text {. }
$$

The numerical eigenvector $\tilde{\phi}_{0}$ also needs to be analysed as it contains information about the local error of the numerical scheme. After normalization by the first (nodal) component $\tilde{\phi}_{0}^{(1)}=1$, a local approximation is reconstructed by combining the nodally exact linear interpolation with the numerical eigenvector $\tilde{\phi}_{0}$ for the bubble functions:

$$
\tilde{p}_{0}(\xi)=l_{0}(\xi)+l_{1}(\xi) \mathrm{e}^{\mathrm{i} k(1+\xi) h / 2}+\sum_{q=2}^{P} \tilde{\phi}_{0}^{(q)} l_{q}(\xi),
$$

where $\tilde{p}_{0}$ does not depend on the element number. We can define the amplitude error which measures the difference between the numerical approximation provided by the bubble functions and the exact 
plane wave solution $p_{\text {exact }}=\mathrm{e}^{\mathrm{i} k x}$ :

$$
E_{a}=\frac{\left\|\tilde{p}_{0}-p_{\text {exact }}\right\|_{H^{1}}}{\left\|p_{\text {exact }}\right\|_{H^{1}}}, \quad \text { with }\|f\|_{H^{1}}=\left(\int_{E_{n}}\left(|f|^{2}+\left|f^{\prime}\right|^{2} / k^{2}\right) \mathrm{d} x\right)^{1 / 2},
$$

where the integral in the norm is computed using Gaussian quadrature with a large number of Gauss points. In practice the dispersion and amplitude error will add up at any point on the mesh. But there is a fundamental difference between the two in that the dispersion error tends to accumulate as a wave propagates from the source to the observer (this is the pollution effect) while the amplitude error contributes uniformly on the mesh. As a consequence, when the computational domain contains many wavelengths, the dispersion error is likely to dominate.

In many dispersion analysis, results are presented as a function of the number of elements per wavelength. When considering high-order interpolation, it is generally more relevant to discuss results in terms of the number of degrees of freedom per wavelength which can be defined as

$$
d_{\lambda}^{ \pm}=\frac{2 \pi P}{\left|k^{ \pm}\right| h},
$$

for the upstream $\left(d_{\lambda}^{-}\right)$and the downstream $\left(d_{\lambda}^{+}\right)$waves. Note that these parameters include the change in acoustic wavelength induced by the mean flow. The $d_{\lambda}^{+}$and $d_{\lambda}^{-}$criteria are useful to study independently the upstream and the downstream cases. However, for problems of practical relevance, upstream and downstream waves coexist in the solution and the choice of grid resolution should be based on the shortest possible wavelength, i.e. the upstream wave. Therefore the parameter

$$
d_{\lambda}^{*}=d_{\lambda}^{-}=\frac{2 \pi P}{\left|k^{-}\right| h},
$$

representing the number of degrees of freedom per shortest wavelength is introduced. It will be used both for upstream and downstream waves. In practice, when designing finite element meshes for convected wave propagation, one should prescribe a minimal value for $d_{\lambda}^{*}$. The choice of the most appropriate criterion will be further discussed in Section 3.3. It should be mentioned that the number of degrees of freedom per wavelength does not necessarily provide a complete picture of the computational costs of a numerical method. When solving a given problem within a specified accuracy, higher-order interpolating shape functions generally lead to smaller matrices. However, these matrices are generally more costly to assemble, and exhibit a larger bandwidth and a higher condition number. For these reasons, to reach a target accuracy on a given problem, decreasing the number of degrees of freedom does not necessarily yield a lower computational cost and a trade-off between efficiency and interpolation order has to be achieved [19]. The effect of the mean flow on the condition number of the p-FEM system matrices will be commented in Sec. 4.2. Other performance aspects (run time and memory requirement) will not be considered in the current paper but will be systematically examined on large-scale, three-dimensional problems in a forthcoming study.

\subsection{Discussion of results}

Results will be presented here for the wave travelling in the positive direction with wavenumber $k=k^{+}=\omega /(1+M)$. So $M>0$ corresponds to downstream propagation and $M<0$ to upstream propagation.

Dispersion error: Ihlenburg and Babuška [13] derived an upper bound on the dispersion error $E_{d}$ for the $h p$-FEM for the Helmholtz equation. Ainsworth [15] then obtained various approximations of $E_{d}$ for small or large wavenumbers. By solving the numerical dispersion relation (28), and by comparing the results with Ainsworth's analysis, we have found that the dispersion error of the $p$-FEM for the convected Helmholtz equation can be described by

$$
E_{d}=\frac{1-M}{2}\left[\frac{P !}{(2 P) !}\right]^{2} \frac{(k h)^{2 P}}{2 P+1}+\mathcal{O}(k h)^{2 P+2} \quad \text { when } k h \rightarrow 0,
$$



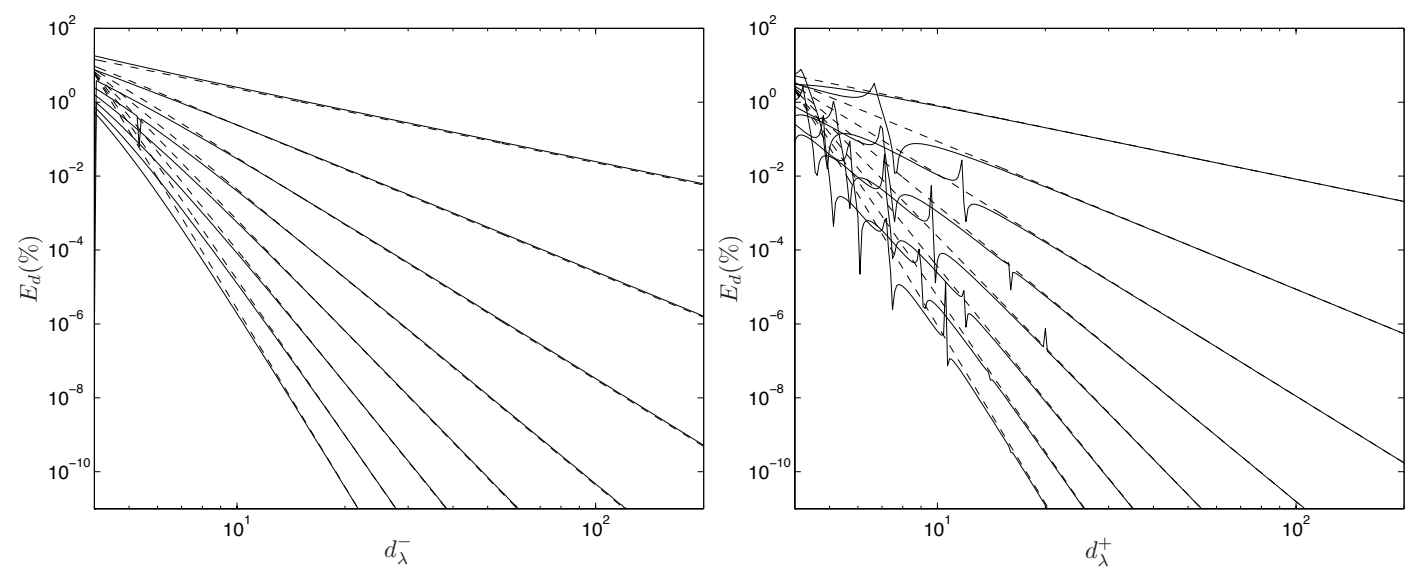

Figure 3. Dispersion error in $\%$ for upstream propagation $M=-0.5$ (left) and downstream propagation $M=0.5$ (right) for a polynomial order $P$ varying from 1 to 8 . Solid lines: numerical dispersion error. Dashed lines: dispersion error predicted by equation (35).

which is the same result as equation (3.4) in [15] except for the additional $1-M$ factor and for the fact that the effect of the mean flow is also accounted for by the relation $k=\omega /(1+M)$. Comparisons of equation (35) with the results obtained by solving the numerical dispersion relation (28) are shown in figure 3. It is clear that equation (35) provides a very accurate approximation of the dispersion error over the whole range of polynomial orders and Mach numbers (with the exception of the aliasing problem which is discussed below). We verified that Equ. (35) is consistent with the dispersion relation for $P=1$ and $P=2$ which can be easily derived and solved in closed form. It was also verified that the next term in (35) is indeed of order $\mathcal{O}(k h)^{2 P+2}$.

It follows that the convergence rate of the dispersion error observed for the Helmholtz equation $\left(E_{d} \sim(k h)^{2 P}\right.$ for $k h$ sufficiently small) remains unchanged with flow. The key property of exponential convergence in $P$ is therefore maintained.

However, the presence of the $1-M$ factor indicates that, even when the change in wavenumber $k$ due to the mean flow is accounted for, the dispersion error increases for upstream waves and decreases for downstream waves. This can be attributed to the convection terms present in the convected wave equation (1). In the worst case $(M \rightarrow-1)$ this corresponds to doubling the dispersion error.

Overall, the mean flow introduces a factor $(1-M) /(1+M)^{2 P}$ in the dispersion error (for fixed frequency and mesh resolution), which is a combination of the change in wavelength introduced by the mean flow and of the $1-M$ factor noted above.

Amplitude error: The amplitude error $E_{a}$ as defined in (32) is also analysed for the downstream and upstream propagations. In either case, by inspection of the numerical results, it is found to behave asymptotically like

$$
E_{a} \simeq C_{a}(P)(k h)^{P}, \quad \text { when } k h \rightarrow 0,
$$

for $P>1$, where $C_{a}$ is a weak function of $P$. Not shown here is the amplitude error measured in terms of the $L_{2}$ norm which was found to have a convergence rate of $\mathcal{O}(k h)^{P+1}$ when $k h \rightarrow 0$.

The amplitude error (32) is plotted for the cases $M= \pm 0.5$ in Figure 4. The main observation is that the asymptotic behaviour of $E_{a}$ is the same for the upstream and downstream waves (in other words $C_{a}(P)$ does not depend on the sign of $M$ ). This is to be expected since $E_{a}$ is a measure of how well the bubble shape functions can interpolate the waveform $\mathrm{e}^{\mathrm{i} k x}$ within each element.

Aliasing: The dispersion and amplitude errors $E_{d}$ and $E_{a}$ are characterized by the presence of numerous error peaks in the low resolution regime when the wave propagates downstream (see figures 3 and 4). The occurrence of these errors peaks corresponds to the so-called aliasing effect [11]. To describe this effect in more detail, let us examine the variational formulation (2) and in 

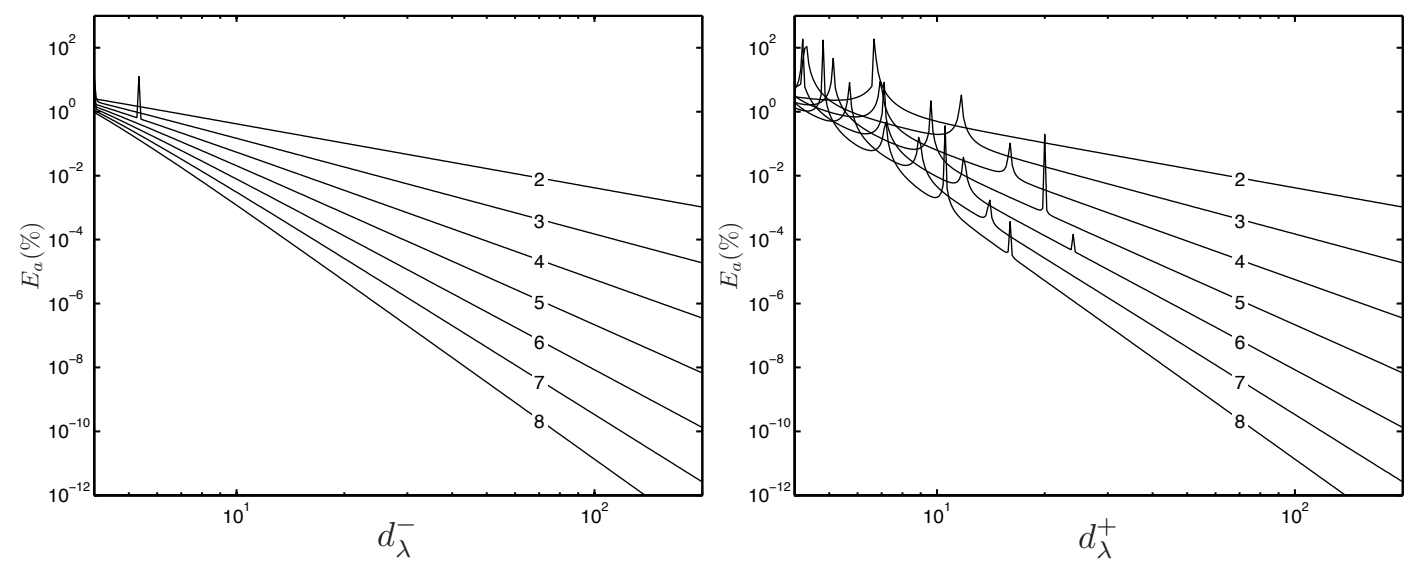

Figure 4. Amplitude error $E_{a}$ in $\%$ for upstream propagation $M=-0.5$ (left) and downstream propagation $M=0.5$ (right) for a polynomial order $P$ varying from 2 to 8 .

particular the part of this formulation governing the bubble degrees of freedom, which can be fully determined at the element level since these shape functions do not interact with neighbouring elements. After separating the nodal and bubble shape functions for both the solution and the test function, we can isolate the variational formulation associated with the bubble functions:

$$
\int_{E_{n}}\left(-\frac{\overline{\mathrm{d}_{0} w_{n}^{b}}}{\mathrm{~d} t} \frac{\mathrm{d}_{0} \tilde{p}_{n}^{b}}{\mathrm{~d} t}+\nabla \overline{w_{n}^{b}} \cdot \nabla \tilde{p}_{n}^{b}\right) \mathrm{d} x=\int_{E_{n}}\left(\overline{\frac{\mathrm{d}_{0} w_{n}^{b}}{\mathrm{~d} t}} \frac{\mathrm{d}_{0} \tilde{p}_{n}^{v}}{\mathrm{~d} t}-\nabla \overline{w_{n}^{b}} \cdot \nabla \tilde{p}_{n}^{v}\right) \mathrm{d} x, \quad \forall w_{n}^{b},
$$

where $w_{n}^{b}$ is the test function associated with the bubble degrees of freedom. The right-hand side can be interpreted as the forcing induced by the nodal degrees of freedom on the bubble shape functions. The operator on the left-hand side can be inverted to statically condense out the bubble degrees of freedom within each element. This is however only possible away from the eigenvalues of this operator, as indicated in $[12,13,15]$. If the parameters (element size, frequency, etc.) correspond to an eigenvalue then the amplitudes of the bubble functions are undefined. The root cause of the aliasing effect is therefore that the bubble functions, which have the benefit of enabling static condensation, introduce the possibility for the corresponding matrix to be singular or poorly conditioned.

To proceed further note that (37) corresponds to the formulation of a simple propagation problem in a one-dimensional acoustic cavity of length $h$. The solution inside this cavity satisfies the convected Helmholtz equation (1) with homogeneous Dirichlet boundary conditions at $x=0$ and at $x=h$. The corresponding eigenvalue problem leads to the following characteristic equation

$$
\mathrm{e}^{\mathrm{i} k^{+} h}=\mathrm{e}^{\mathrm{i} k^{-} h},
$$

which defines the modes of the cavity. Applying the static condensation is equivalent to solving a small Dirichlet problem inside each element describing the acoustic field in a cavity. The aliasing effect can hence be interpreted as spurious internal resonances within each element.

Equation (38) also indicates that at the nodes of the mesh the left-running wave is indistinguishable from the right-running wave (that is $\exp \left(\mathrm{i} k^{+} x_{m}\right)=\exp \left(\mathrm{i} k^{-} x_{m}\right)$ with $\left.x_{m}=m h\right)$, hence justifying the name 'aliasing effect' used by Mulder [11].

Using (29), we find that for the convected wave equation the aliasing effect occurs for the following values of the number of degrees of freedom per downstream wavelength $d_{\lambda}^{+}$or degrees of freedom per shortest wavelength $d_{\lambda}^{*}$ :

$$
d_{\lambda}^{+}=\frac{2 P}{j(1-M)}, \quad d_{\lambda}^{*}=\frac{2 P}{j(1+M)},
$$

with $j$ a strictly positive integer. It is worth noting that these only constitute approximations since they are based on the theoretical dispersion relation (29). It can also be noted that the more accurate 


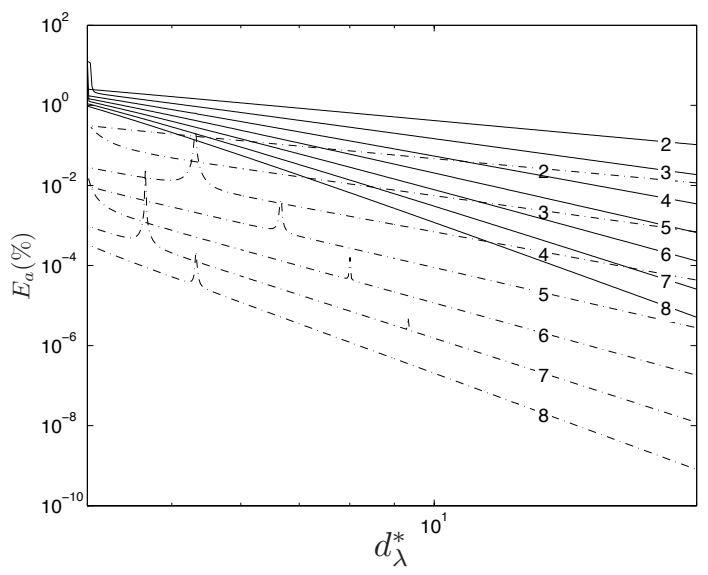

Figure 5. Absolute amplitude error $E_{a}$ in $\%$ as a function of the number of degrees of freedom per shortest wavelength $d_{\lambda}^{*}$ for a polynomial order $P$ varying from 2 to 8 . Solid lines: Upstream case $(M=-0.5)$. Dot-dashed lines: Downstream case $(M=0.5)$.

the numerical model is, the more localized the aliasing effect becomes, that is: for high resolutions, the width of each aliasing peak becomes narrower, as seen in figure 4.

The remaining question is that of the significance of the aliasing error in practical situations. In the absence of flow $(M=0)$, Equ. (39) indicates that aliasing effects occur when the element size equals at least half an acoustic wavelength. Such a mesh resolution is not used in practice for loworder elements (say $P<3$ ) but may occur for higher-order elements which typically span a larger portion of a wavelength. In Ref. [11] aliasing peaks are observed at common discretization rates but it is however concluded that their impact on the global numerical error is limited.

In the presence of downstream flow, Equation (39) indicates that the aliasing effect is even emphasized, because the aliasing error peaks migrate to higher mesh resolutions. In reference [20] it was therefore concluded that the aliasing has an important impact for convected applications and should lead to finer mesh requirements. In the present paper, we qualify this interpretation by showing that the aliasing error is in fact of limited significance in practical applications. The difference in interpretation lies in the choice of the criterion used to measure the resolution rate. In the presence of flow, the aliasing effect does indeed move to higher mesh resolutions when measured using $d_{\lambda}^{+}$, but it also corresponds to low resolutions when measured using $d_{\lambda}^{*}$, as indicated by equation (39). One has to keep in mind that in practice the grid is designed based on the shortest possible wavelength, that is based on $d_{\lambda}^{*}$. For instance, in the case $M=0.5$ we have $d_{\lambda}^{*}=d_{\lambda}^{+} / 3$, the region in which the aliasing peaks dominate $E_{d}$ and $E_{a}$ in the downstream case corresponds to a region in which the upstream wave is clearly under-resolved.

In Figure 4, the amplitude error $E_{a}$ is plotted as a function of $d_{\lambda}^{+}$or $d_{\lambda}^{-}$for the upstream and downstream cases, respectively. In Figure 5, the same results are plotted together as a function of $d_{\lambda}^{*}$. This provides a more representative description of the link between the choice of mesh resolution and the impact of the aliasing effect. Even at the error peaks, the amplitude errors on the downstream wave are a few orders of magnitude lower than the errors incurred on the upstream wave. This indicates that the numerical error will be driven by the discretization of the upstream wave. Provided that the mesh resolutions are always defined in terms of the shortest wavelength using $d_{\lambda}^{*}$, the dispersion analysis shows that the loss of accuracy due to the aliasing effect is offset by the fact that the downstream wave is necessarily over-resolved. This conclusion is also supported by the results of the test case presented in the next section.

\subsection{Application to a simple test case}

We now consider a simple one-dimensional test case to assess the global numerical error observed in practice, and to discuss the effect of the mean flow and of the aliasing effect on the performance of 
the $p$-FEM. Three different types of grids are studied (uniform, periodic and random, as illustrated in figure 6).

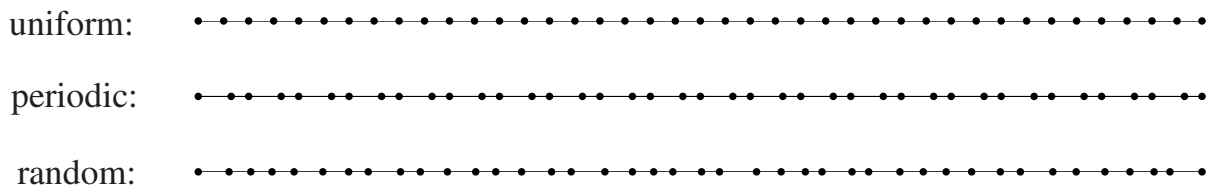

Figure 6. Meshes used for the one-dimensional test case with 40 elements.

3.4.1. Uniform grid Let us first consider a uniform grid of length $L$ and with elements of size $h$. The Robin-type boundary condition (3) is used at both ends of the computational domain, with $g=-2 \mathrm{i} k$ at $x=0$ and $g=0$ at $x=L$ so as to create a single propagating wave $p_{\text {exact }}=\mathrm{e}^{\mathrm{i} k x}$ in the computational domain. We calculate the relative error measured in $H^{1}$ norm

$$
E_{1}=\frac{\left\|p-p_{\text {exact }}\right\|_{H^{1}}}{\left\|p_{\text {exact }}\right\|_{H^{1}}} .
$$

Figure 7 shows $E_{1}$ as a function of the number of degrees of freedom per wavelength $d_{\lambda}^{+}$or $d_{\lambda}^{-}$when the wave is going with the flow (downstream propagation $M>0$ ) or against the flow (upstream propagation $M<0$ ).

Error estimates: For the $h p$-FEM for the Helmholtz equation it has been shown that $E_{1}$ can be estimated by $[26,13]$ :

$$
E_{1} \leqslant C_{1}\left(\frac{k h}{2 P}\right)^{P}+C_{2} k\left(\frac{k h}{2 P}\right)^{2 P},
$$

where $C_{1}$ and $C_{2}$ are independent of $\omega$ and $h$, and are weak functions of $P$. It is worth noting that the number of degrees of freedom per wavelength is inversely proportional to $(k h) /(2 P)$. By comparing with the numerical error measured in the test case described above, it was found that for the convected Helmholtz equation (1) the numerical error remains well approximated by (40).

The first term in (40) represents the asymptotic behavior of the numerical error $(k h \rightarrow 0)$ and is associated with the quality of the best interpolation. The presence of the mean flow does not modify this asymptotic behavior: the $E_{1}$ error decays like $(k h)^{P}$ (and the relative error in $L^{2}$-norm like $(k h)^{P+1}$ ). In addition, we find that $C_{1}$ remains independent of the Mach number $M$ and the size of the domain $L$. This is expected since the first term in (40) is associated with the approximation error and the effect of the mean flow on the wavelength is already fully accounted for by the definition $k=\omega /(1+M)$. This is consistent with the analysis of the amplitude error in the dispersion analysis in Section 3.3.

The $h p$-FEM for the Helmholtz equation is quasi-optimal, i.e. in the asymptotic range $(h \rightarrow 0)$ the numerical error is proportional to the error associated with the best approximation, see equation 11 in [26]. With flow we find that the numerical model remains quasi-optimal and the optimality constant $C_{\mathrm{opt}}$ is independent of the mean flow velocity. For polynomial orders of up to 10 we observe that $1 \leqslant C_{\mathrm{opt}} \leqslant 1.3$ when $h \rightarrow 0$ indicating that in the asymptotic range the FE model yields solutions close to the best approximations.

The second term in equation (40) is the pollution error which is driven by the build-up of phase lead due to the dispersion error (35) as the wave propagates. It becomes significant for high frequencies, or when the computational model contains many wavelengths.

It was found that the parameter $C_{2}$, in addition to being a function of the interpolation order, is a function of the flow Mach number $M$ and the domain length $L$. The dependence on $L$ is expected because the longer the domain the more the dispersion error will be able to build up as the wave propagates from $x=0$ to $x=L . C_{2}$ also scales with $(1-M)$, which is consistent with the observation made in Section 3.3 that the dispersion error (35) has an additional $(1-M)$ factor when flow is present. This is illustrated in figure 8 where the error $E_{1}$ scaled by $(1-M) L$ is plotted, 

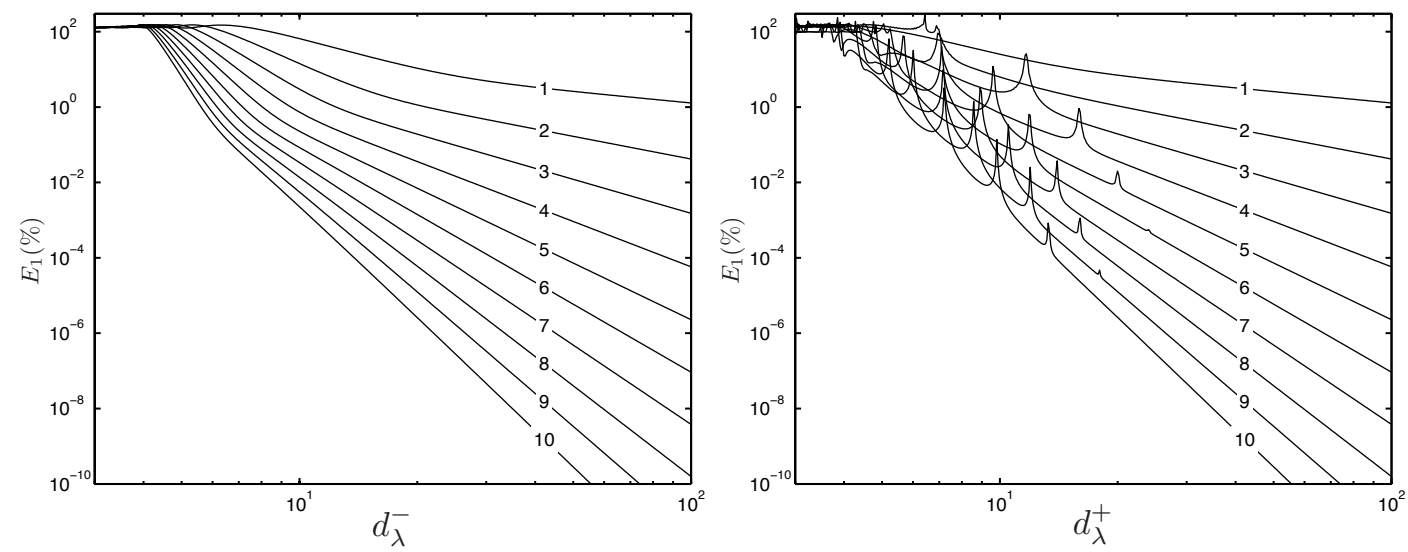

Figure 7. Numerical error $E_{1}$ in $\%$ as a function of the number of degrees of freedom per wavelength (with $h$ fixed and $\omega$ varying), with $L=80, h=1$ and for a polynomial order $P$ varying from 1 to 10 . Left: wave propagating upstream $M=-0.5$. Right: wave propagating downstream $M=0.5$.
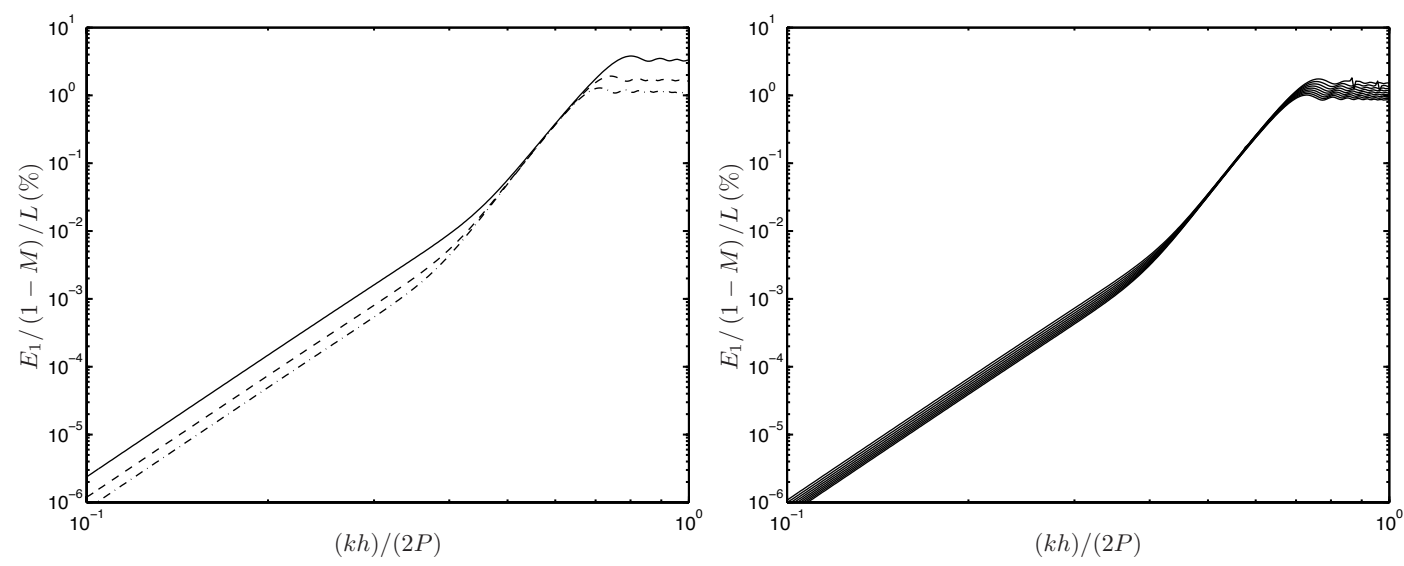

Figure 8 . Numerical error $E_{1}$ scaled by $(1-M) L$ and plotted against $(k h) /(2 P)$ for fixed polynomial order $P=6$ and with $h=1$. Left: Fixed Mach number $M=-0.5$ with domain size $L=40$ (solid line), 80 (dashed line) and 120 (dot-dashed line). Right: Fixed domain size $L=80$ with Mach number varying from $M=0.0$ to -0.9 with a step of 0.1 .

either by varying the domain size ( $L=40,80$ and 120) with a fixed Mach number, or by varying the Mach number with a fixed domain size $(L=80)$. It can be seen that the convergence plots collapse onto the same curve in the region where the dispersion error dominates the global numerical error $E_{1}$, clearly indicating that the second term in (40) scales like $(1-M) L$.

Therefore, the presence of the mean flow can degrade the performance of the FE model at high wavenumbers (that is in the pre-asymptotic range when the dispersion error dominates the numerical error). Again, this can be attributed to the additional convection terms in the wave operator (1) that tend to reduce the accuracy of the FE approximation.

Aliasing: Equation (40) does not capture the aliasing effect. In fact it is only valid when $k h<\pi$ which precisely excludes aliasing [13]. The aliasing peaks visible in the downstream case in figure 7 can however be closely approximated by equation (39). As explained in section 3.3, to assess the effect of the aliasing effect it is more representative of engineering practice to use $d_{\lambda}^{*}$ to measure the mesh resolution. This is done in figure 9 where the peaks of error for the downstream waves are still visible but correspond to much lower levels of error compared to the upstream waves. So in practice the aliasing effect can occur only when the upstream wave is under-resolved.

Also noticeable for the no flow case are the very small increases of error associated with the aliasing when the element size is a multiple of half the wavelength. Aliasing errors are therefore 


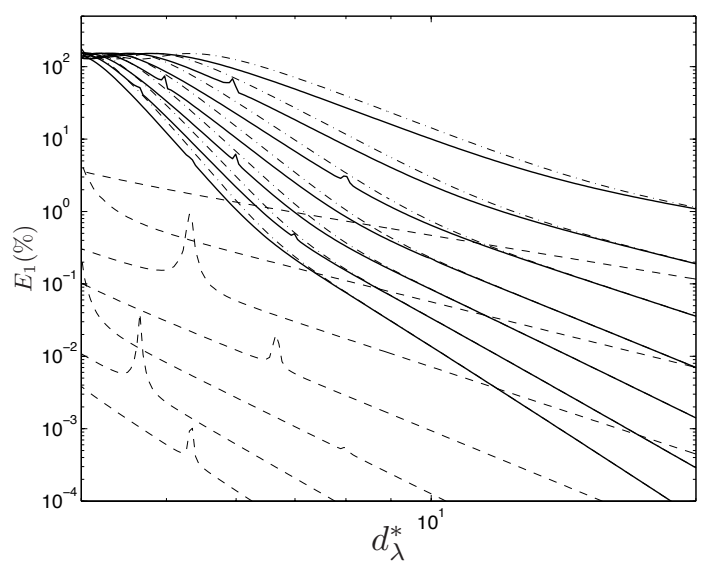

Figure 9. $E_{1}$ error obtained with the uniform mesh with domain size $L=80$ for a polynomial order from $P=2$ to 8 . Solid line: no-flow case $(M=0)$. Dot-dashed line: upstream case $(M=-0.5)$. Dashed line: downstream case $(M=0.5)$.
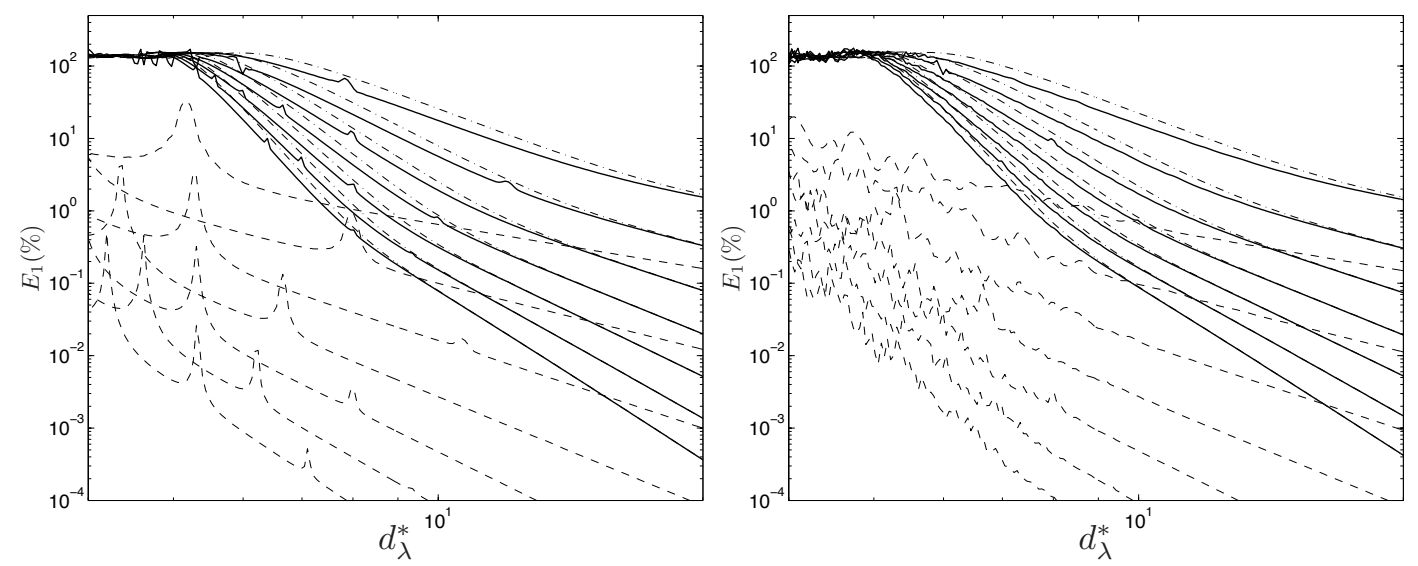

Figure 10. $E_{1}$ error obtained with the periodic mesh (left) and the random mesh (right) with domain size $L=80$ for a polynomial order from $P=2$ to 8 . Solid line: no-flow case $(M=0)$. Dot-dashed line: upstream case $(M=-0.5)$. Dashed line: downstream case $(M=0.5)$

also influencing the solution in the no-flow case at common discretization rates. However, the amplitude of the peaks is much smaller than in the presence of flow. The impact of the aliasing on the performance of the Helmholtz equation is therefore also very limited. This is in line with the previous conclusions of Mulder [11].

3.4.2. Unstructured grid In practice meshes are rarely uniform so we consider two non-uniform grids, as depicted in figure 6, with a periodic mesh with a two-element pattern of consecutive element sizes $h_{1}=1.3$ and $h_{2}=0.7$, and a mesh with randomized element sizes. The domain length is $L=80$ and the number of elements is kept constant so that the resulting computational effort remains equivalent for all meshes. In the definitions of the number of degrees of freedom per wavelength (33)-(34), the averaged value of the mesh size $h=1$ is used. The $E_{1}$ errors obtained on the periodic and the random meshes are shown for $M=0, M=0.5$ and $M=-0.5$ in figure 10 as a function of $d_{\lambda}^{*}$.

The overall behavior of the numerical error is similar with that observed with a uniform mesh. A key difference is the occurrence of the peaks associated with the aliasing effect. Equations (34) and (39) can be combined to show that the angular frequency required for an internal resonance within a element of size $h$ is given by $\omega=j \pi\left(1-M^{2}\right) / h$. So each element will exhibits internal resonances at different frequencies. This explains the multiplicity of error peaks observed in figure 10. But it 


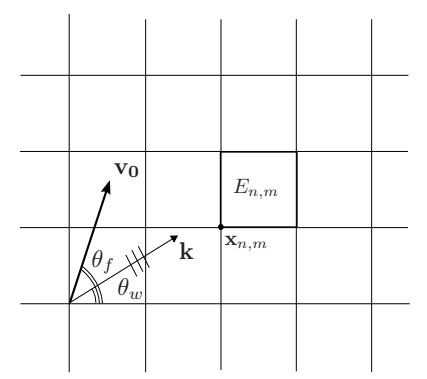

(a) Quadrangle mesh

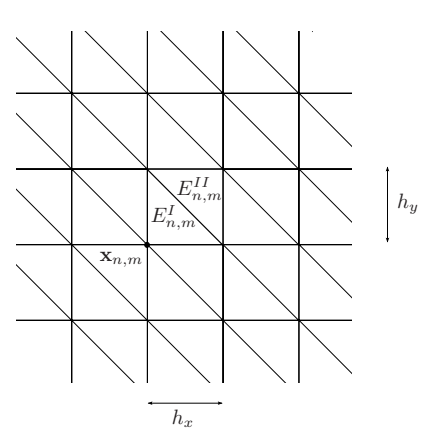

(b) Triangle mesh $\mathrm{A}$

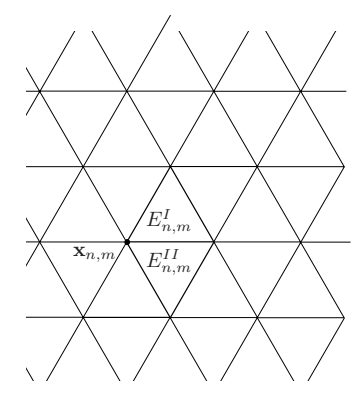

(c) Triangle mesh B

Figure 11. Finite element meshes used for the two-dimensional dispersion analysis.

can be noticed as well that the magnitude of these peaks is somewhat reduced in the case of the random mesh.

\section{TWO-DIMENSIONAL DISPERSION ANALYSIS}

We now move on to the case of two-dimensional meshes, with the aim to assess the effect of the mesh topology and to see if the observations made for one-dimensional problems remain applicable.

We consider three infinite periodic meshes, one with quadrangle elements and two with triangular elements, as shown in Figure 11. The meshes are periodic in the sense that they are composed of a 'stencil' of element(s), denoted $E_{n, m}$, which is repeated in all directions to build the infinite mesh. On the quadrangle mesh, the stencil is composed of a single element, whereas it is composed of two elements on the triangular meshes. Each element stencil is characterized by its origin, denoted $\mathbf{x}_{n, m}=\left(n h_{x}, m h_{y}\right)$ for the quadrangle mesh and for the triangle mesh $\mathrm{A}$, and $\mathbf{x}_{n, m}=$ $\left(n h_{x}+m h_{x} / 2, m h_{y}\right)$ for the triangle mesh B. The dispersion properties of similar mesh topologies have already been studied in the no-flow case for conventional low-order finite elements by Harari et al. [27] and for high-order elements by Deraemaeker et al. [14]. Both studies demonstrated the strong influence of the mesh topology on the performance of the numerical method.

We seek plane wave solutions

$$
p(x, y)=\mathrm{e}^{\mathrm{ik} \cdot \mathbf{x}},
$$

with the exact wavenumber $\mathbf{k}$ given by

$$
\mathbf{k}=\left(k_{x}, k_{y}\right)=\left(k \cos \theta_{w}, k \sin \theta_{w}\right), \quad \text { with } k=\frac{\omega}{1+M \cos \left(\theta_{w}-\theta_{f}\right)} .
$$

where $M=\left|\mathbf{v}_{0}\right| / c_{0} . \theta_{w}$ and $\theta_{f}$ denote the wave angle and the orientation of the mean flow, respectively. The discretization of the weak form (2) using $p$-FEM elements on the periodic meshes yields an algebraic system of infinite size, of the form (18). The vector of unknowns $\phi_{\infty}$ now contains nodal, edge and bubble degrees of freedom. Following the approach presented in the onedimensional case, it can be shown that these unknowns belong to a finite number $N$ of classes such that all degrees of freedom of a given class satisfy the same difference equations. A total of $N=P^{2}$ classes are identified for the three meshes. Once the classes are identified, the dispersion relation of the numerical model (28) can be readily obtained. The complete demonstrations are given in Appendix A for the quadrangle mesh and in Appendix B for the two triangular meshes. For a given angular frequency $\omega$, mesh size $\left(h_{x}, h_{x}\right)$, wave orientation $\theta_{w}$ and flow direction $\theta_{f}$, a discrete wavenumber $\tilde{k}$ is obtained by solving the system (28), and compared to the exact dispersion relation given in Equation (42) to obtain the dispersion error $E_{d}$, as defined by Equation (30).

To compute the amplitude error, the numerical approximation $\tilde{p_{0}}$ is first reconstructed from the numerical eigenvector $\tilde{\phi}_{0}$. After normalization by the first (nodal) component $\tilde{\phi}_{0}^{(1)}=1$, it takes the 
following form for the quadrangle

$$
\tilde{p_{0}}(\xi, \eta)=\sum_{r=1}^{4} \varphi^{v_{r}}(\xi, \eta) \mathrm{e}^{\mathrm{i} \mathbf{k} \cdot \mathbf{x}_{r}}+\sum_{g=2}^{N} \varphi^{g}(\xi, \eta) \tilde{\phi}_{0}^{(g)},
$$

where $\varphi^{v_{r}}$ designates the standard bilinear shape functions. $\mathbf{x}_{r}$ corresponds to the node location on the grid. $\tilde{\phi}_{0}^{(g)}$ is the amplitude of a given edge or bubble degree of freedom given in Appendix A and $\varphi^{g}(\xi, \eta)$ is the corresponding shape function. Note that $\tilde{p_{0}}$ does not depend on the element number. The amplitude error measures the difference between this numerical approximation and the exact plane wave solution $p_{\text {exact }}=\mathrm{e}^{\mathrm{i} \mathbf{k} \cdot \mathbf{x}}$ on an element stencil $E_{n, m}$, as follows,

$$
E_{a}=\frac{\left\|\tilde{p_{0}}-p_{\text {exact }}\right\|_{H^{1}}}{\left\|p_{\text {exact }}\right\|_{H^{1}}}, \quad\|f\|_{H^{1}}=\left(\int_{E_{n, m}}\left(|f|^{2}+|\nabla f|^{2} / k^{2}\right) \mathrm{d} \mathbf{x}\right)^{1 / 2} .
$$

Let us now define some indicators for the resolution effort. When solving a boundary value problem on a two-dimensional bounded computational domain $\Omega$, the number of degrees of freedom per wavelength is generally defined as follows

$$
d_{\lambda}=\sqrt{\frac{N_{\mathrm{dof}}}{\operatorname{Area}(\Omega)}} \frac{2 \pi}{|k|},
$$

where $N_{\text {dof }}$ and Area $(\Omega)$ denote respectively the total number of unknowns and the domain area. However, it is not so straightforward to define such a criterion on an unbounded domain. Following [14], a 'density of unknowns' is used here, whereby weighting coefficients of $1 / 4,1 / 2$ and 1 are associated with nodal, edge and bubble degrees of freedom, respectively. These coefficients correspond to the area covered by each type of shape functions. On an element stencil $E_{n, m}$, this yields a density of unknowns of $N_{\text {dof }}=\left(\frac{1}{4} \times 4\right)+\frac{1}{2} \times 4(P-1)+1 \times(P-1)^{2}$, which simply leads to $N_{\text {dof }}=P^{2}$. The following definition is therefore introduced:

$$
d_{\lambda}=\frac{2 \pi}{|k| \sqrt{h_{x} h_{y}}} P .
$$

For the sake of conciseness, only square stencils are considered in this study, such that $h_{x}=h_{y}=$ $h$. In this case, definition (46) is equivalent to the one derived for the one-dimensional dispersion analysis (33). The number of degrees of freedom per shortest wavelength $d_{\lambda}^{*}$ is also defined by considering the wave propagating against the flow $\left(\theta_{w}=\theta_{f}+\pi\right)$ :

$$
d_{\lambda}^{*}=\frac{2 \pi(1-M)}{\omega h} P .
$$

Note that in the no flow case, the two parameters are equivalent. In Ref. [21], it is shown that the mean flow orientation has only a marginal impact on the properties of the numerical scheme in comparison with the other parameters, such as the Mach number and the wave orientation. Therefore, for the sake of simplicity, only the case $\theta_{f}=0$ will be considered throughout this study. Besides, when the wave propagates along the lines of the mesh, the results are very similar to the ones obtained in the one-dimensional case. Therefore, the analysis will focus on the anisotropy properties of the dispersion and amplitude errors with the objective to determine the influence of the wave propagation angle on the accuracy for flow acoustics with different mesh topologies.

\subsection{Discussion of results}

To study the anisotropy properties of the $p$-FEM on the different mesh stencils, a fixed resolution of 6 degrees of freedom per wavelength is used. The Mach number is fixed at $M=0.5$. Two representations are proposed, one using $d_{\lambda}=6$, similar to the one used in Reference [20] where the mesh size $h$ is adjusted to the plane wave orientation at each angle, or by setting $d_{\lambda}^{*}=6$ where 

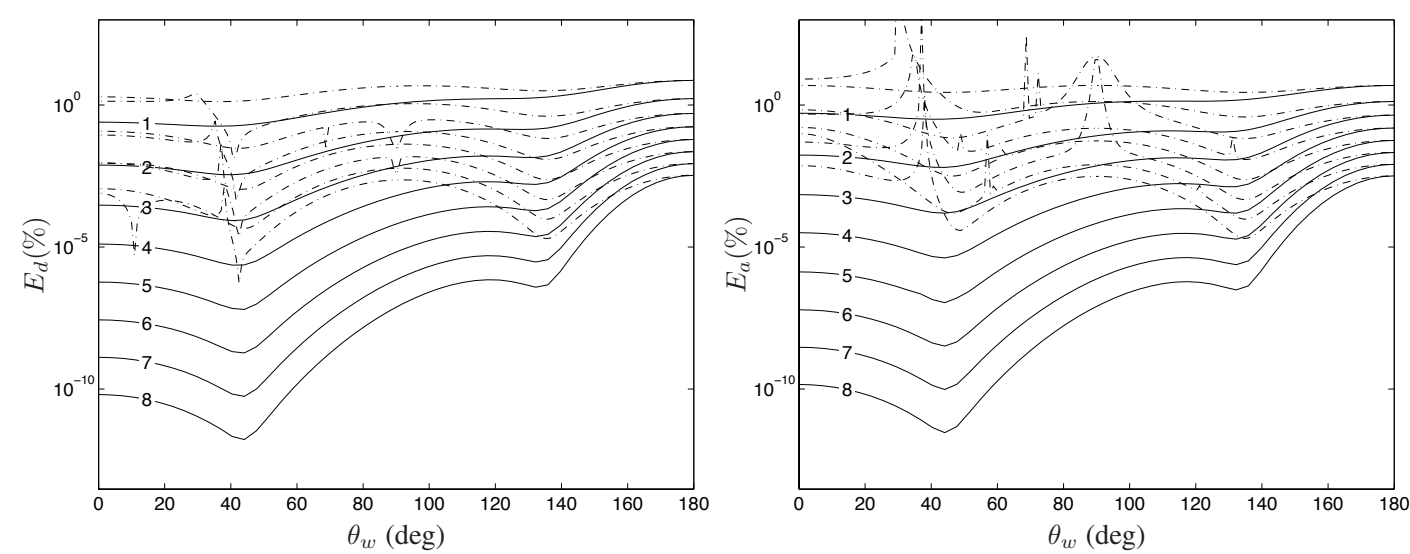

Figure 12. Dispersion error $E_{d}$ (left) and amplitude error $E_{a}$ (right) in $\%$ for $M=0.5$ on the quadrangle as a function of the propagation angle $\theta_{w}$, for a polynomial of order $P$ varying from 1 to 8 with a step of 1 . Solid lines: $d_{\lambda}^{*}=6$. Dash-dotted lines: $d_{\lambda}=6$.
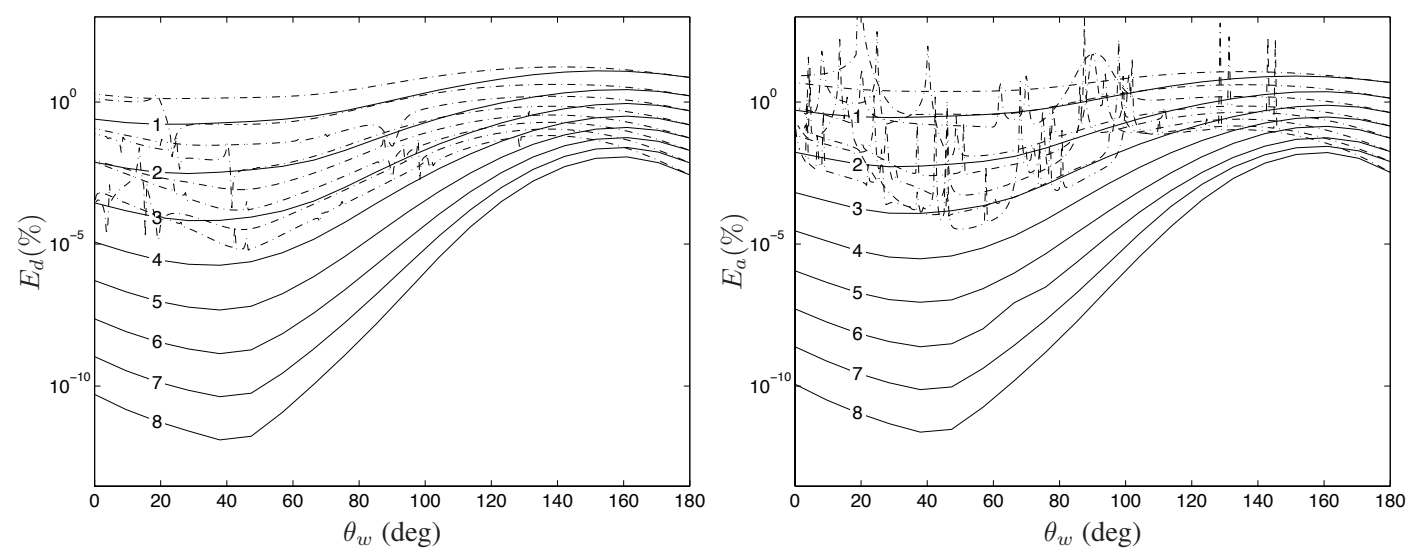

Figure 13. Dispersion error $E_{d}$ (left) and amplitude error $E_{a}$ (right) in $\%$ for $M=0.5$ on the triangle stencil A as a function of the propagation angle $\theta_{w}$, for a polynomial of order $P$ varying from 1 to 8 with a step of 1. Solid lines: $d_{\lambda}^{*}=6$. Dash-dotted lines: $d_{\lambda}=6$.

the mesh size is fixed to ensure that the shortest wavelength (corresponding to the purely upstream wave) is sufficiently resolved. Obviously, for $\theta_{w}=180^{\circ}$, the two criteria are equivalent.

The dispersion and amplitude errors are plotted up to order $P=8$ as a function of the wave propagation angle $\theta_{w}$ in Figures 12,13 and 14 for the quadrangle mesh, the triangle mesh $\mathrm{A}$ and the triangle mesh B respectively. Taking symmetry into account, the results are shown only in the interval $\theta_{w}=\left[0,180^{\circ}\right]$.

When setting $d_{\lambda}=6$, the aliasing peaks completely dominate the amplitude error for low angles $\left(\theta_{w}=\left[0,90^{\circ}\right]\right)$ corresponding to the downstream propagation. The aliasing effect is even more pronounced on the triangle meshes where higher angles are also affected (up to $\theta_{w}=140^{\circ}$ ). The higher-order elements present better approximation properties but, overall, this is mitigated by the aliasing effect. In any case, even at high orders, the robustness of the $p$-FEM cannot be guaranteed using that criterion.

When using the criterion $d_{\lambda}^{*}=6$, aliasing peaks are not visible and the numerical model is much more robust. For downstream propagation $\left(\theta_{w}<90^{\circ}\right)$, very high $p$-convergence is observed. The dispersion and amplitude errors are decreased by more than one order of magnitude when the polynomial order is increased from $P$ to $P+1$, leading rapidly to virtually 'dispersion-free' propagation. For angles close to the upstream propagation, the $p$-convergence is not as strong but still effective. It is important to compare the performance of the different meshes on this particular region that will, most likely, dominate the numerical error on practical applications. 

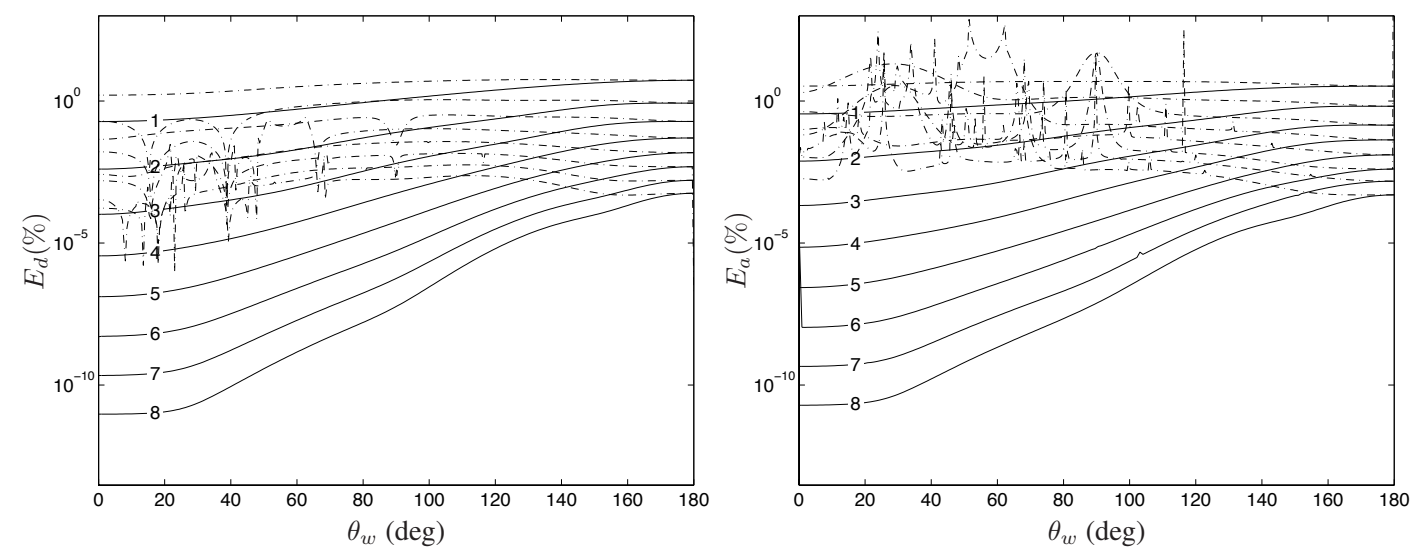

Figure 14. Dispersion error $E_{d}$ (left) and amplitude error $E_{a}$ (right) in $\%$ for $M=0.5$ on the triangle stencil $\mathrm{B}$ as a function of the propagation angle $\theta_{w}$, for a polynomial of order $P$ varying from 1 to 8 with a step of 1. Solid lines: $d_{\lambda}^{*}=6$. Dash-dotted lines: $d_{\lambda}=6$.

- At $\theta_{w}=180^{\circ}$, the triangle mesh A and the quadrangle mesh exhibit the same performance. When the flow and the plane wave are exactly aligned with the mesh nodes, the problem can be considered as purely one-dimensional.

- The triangle mesh A performs poorly in the upstream region $\left(\theta_{w}>90^{\circ}\right)$. Surprisingly enough, the anisotropy is such that the worst performance is not observed for the pure upstream angle $\left(\theta_{w}=180^{\circ}\right)$ but for a substantially lower angle $\left(\theta_{w} \approx 160^{\circ}\right)$. For this particular mesh topology, it may therefore be judicious to adapt the $d_{\lambda}^{*}$ criterion.

- Overall, the triangle mesh B offers the best performance (this is consistent with the observations made in the no-flow case for linear elements by Harari et al. [27]). The anisotropy is less severe and for a given order $P$, the dispersion and amplitude errors are substantially lower than for the two other meshes in the vicinity of $\theta_{w}=180^{\circ}$. For instance, an order $P=4$ on the stencil B yields smaller dispersion and amplitude errors than an order $P=5$ on the two other meshes.

These results confirm the fact that, provided that the waves are sufficiently resolved for all angles of propagation, $p$-FEM is a very efficient scheme for convected acoustics applications. Also, some important anisotropy effects may be observed on periodic meshes.

\subsection{Application test case}

Following the analysis carried out in one dimension, let us now consider a simple two dimensional test case to assess the global numerical error observed in practice. The computational domain $\Omega$ is rectangular with $0<x<50$ and $0<y<10$. We consider structured and unstructured meshes, both with the same numbers of nodes and elements (see Figure 15). A hard-wall condition is defined at $y=0$ and $y=10$, and a Robin-type boundary condition is used at $x=0$ (with $g=-2 \mathrm{i} k$ ) and at $x=50$ (with $g=0$ ) so as to create a wave $p_{\text {exact }}=\mathrm{e}^{\mathrm{i} k x}$ propagating from left to right in the computational domain.

We calculate the relative error measured in $H^{1}$ norm defined as

$$
E_{1}=\frac{\left\|p-p_{\text {exact }}\right\|_{H^{1}}}{\left\|p_{\text {exact }}\right\|_{H^{1}}} .
$$

Figure 16 shows $E_{1}$ as a function of the number of degrees of freedom per shortest wavelength $d_{\lambda}^{*}$ for the case of a wave propagating upstream $\mathbf{v}_{0}=(-0.5,0)$ and downstream $\mathbf{v}_{0}=(0.5,0)$. The results are similar to the ones obtained in the one-dimensional case in Section 3.4, even on the unstructured grid for which the problem is truly bi-dimensional. As a result, the numerical error remains in line with the error estimator (40). In the low resolution regime, where the dispersion error dominates, the presence of the mean flow can degrade significantly the performance of the FE 

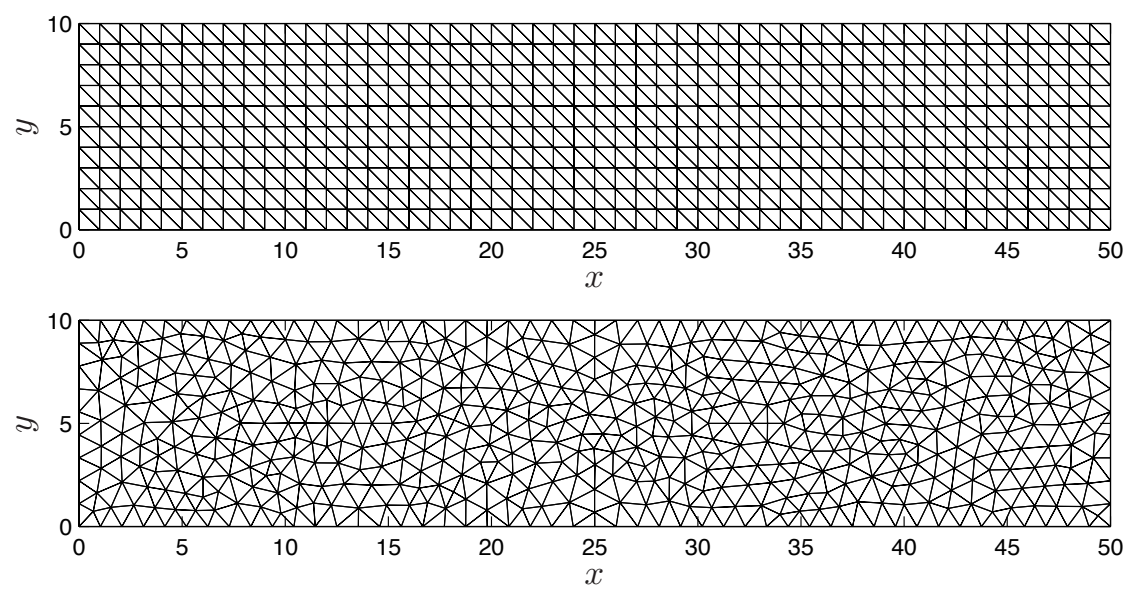

Figure 15. Structured mesh (top) and unstructured mesh (bottom) used for the two-dimensional test case.

model. The downstream wave exhibits numerous aliasing peaks. However their impact on the overall performance is marginal since the global error is dominated by the discretization of the upstream wave. The performances of the two computational grids are very comparable which highlights the ability of the $p$-FEM to effectively deal with unstructured grids. The main difference lies in the number and amplitude of the aliasing peaks, which are reduced when using an unstructured mesh.
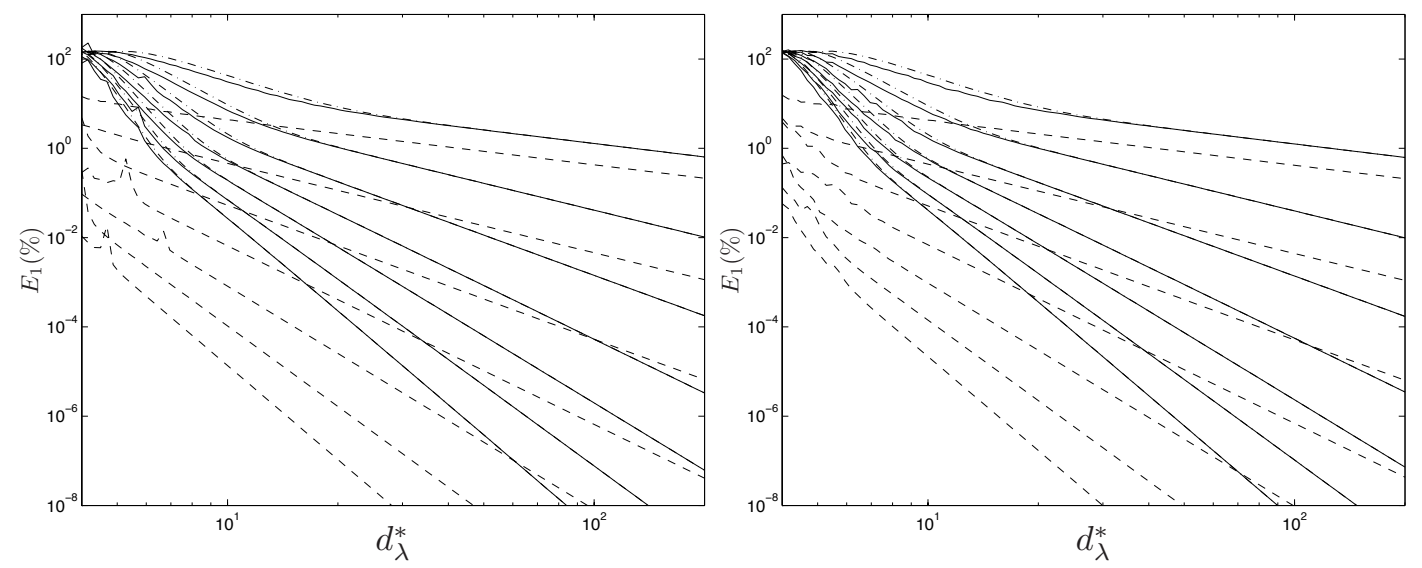

Figure 16. $E_{1}$ error obtained with the structured mesh (left) and the unstructured mesh (right) for polynomial order from $P=2$ to 8 . Solid line: no-flow case. Dashed dotted line: upstream case $(M=-0.5)$. Dashed line: downstream case $(M=0.5)$.

The superiority of the $p$-FEM is also highlighted on this test case. To reach a reasonable engineering accuracy, say $1 \%$ error for $E_{1}$, a mesh resolution as high as $d_{\lambda}^{*}=120$ is required with $P=2$. For $P=8$, this reduces to $d_{\lambda}^{*}=6.3$ in the no flow-case and $d_{\lambda}^{*}=6.5$ at $M=0.5$. This clearly demonstrates the ability of the $p$-FEM to mitigate the pollution effect when solving wave propagation phenomena, including in the presence of strong background mean flow.

It is well known that the condition number of finite element matrices constructed from interpolating basis functions grows as the polynomial order of the basis functions is increased [23]. The poor conditioning of the system matrix can result in slow convergence of iterative solution procedures but may also hinder the stability of a direct solution procedure. In Fig. 17, the condition number of the $p$-FEM system matrix obtained for several orders $P$ is plotted as a function of the mesh resolution measured using $d_{\lambda}^{*}$. An important observation is the fact that the condition number is not deteriorated by the presence of the mean flow. Especially in the asymptotic range, and for the same number of degrees of freedom per wavelength, the no-flow, upstream 
and downstream system matrices yield comparable condition numbers. As expected, the condition number increases regularly with the order of interpolation, and it remains acceptable for the orders studied here $(P \leq 8)$. However, for much higher-orders, say $P<15$, this could limit the practical use of hierarchical basis. For low polynomial orders, the condition number of the global matrix increases with the number of degrees of freedom per wavelength (i.e for low values of the angular frequency $\omega$ ). In contrast, for higher polynomial orders the conditioning is quite poor in the preasymptotic range, where the solution is not satisfactory. Interestingly, in the asymptotic range when the interpolation error dominates, the conditioning appears to be independent of the mesh resolution.
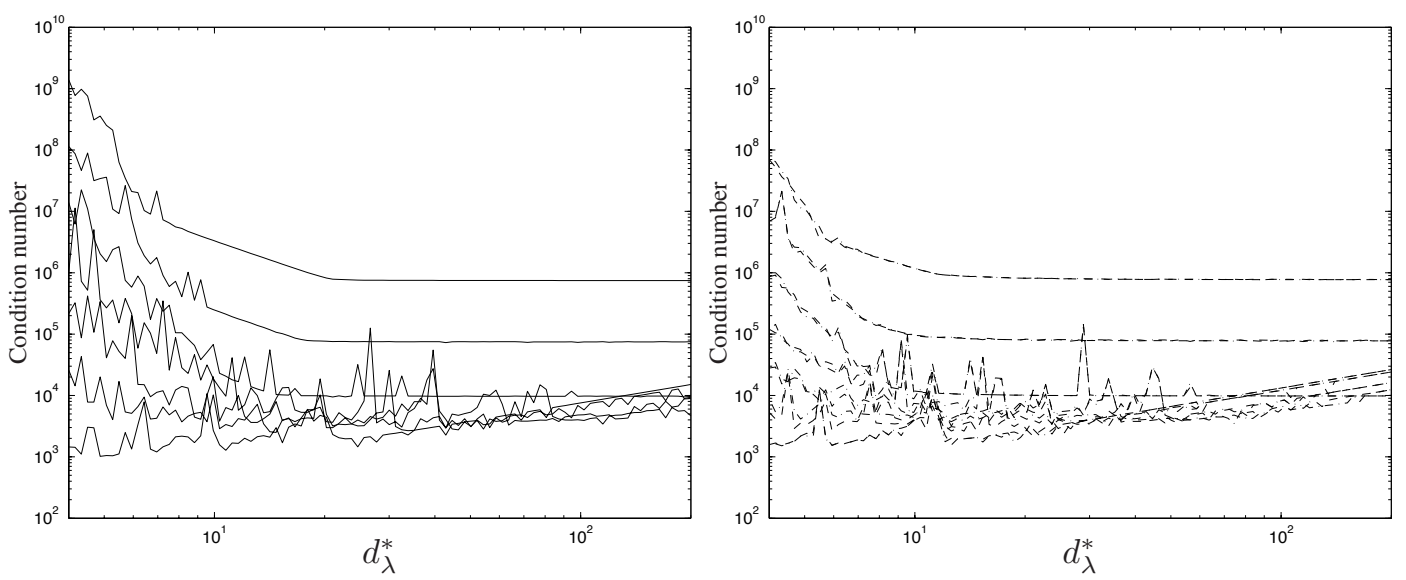

Figure 17. Condition number of the system matrix for the no-flow case (left) and in the presence of flow (right) obtained with the unstructured mesh for polynomial order from $P=2$ to 8 . Solid line: no-flow case. Dashed dotted line: upstream case $(M=-0.5)$. Dashed line: downstream case $(M=0.5)$.

\section{CONCLUSIONS}

An analysis of the performance of the $p$-FEM was presented in the context of convected wave propagation. This assessment was carried out through dispersion analyses in both one and two dimensions to systematically measure the dispersion and amplitude errors of the discrete model. In addition simple benchmark problems with structured and unstrustured meshes have been considered to confirm that the conclusions of the dispersion analyses apply also to actual simulations.

The overall conclusion is that the properties of $p$-FEM that make its strength for standard acoustics (e.g. exponential $p$-convergence) remain present for flow acoustics as well. More precisely, by modifying a result derived by Ainsworth, an estimator for the dispersion error which incorporates convection effects was proposed. It features an additional $(1-M)$ coefficient even when the change in wavelength due to the mean flow is accounted for. In the worst case, this leads to a doubling of the phase errors for waves propagating upstream. At high wavenumbers, where the dispersion error is likely to dominate the total numerical error, the presence of mean flow can degrade the performance of the numerical scheme. However the key property of exponential convergence of the dispersion error with increasing polynomial order $P$ is conserved and as such, $p$-FEM elements prove to efficiently mitigate the pollution effect also for convected applications. The condition number was also examined and it is proved not to be deteriorated by the presence of mean flow. Other performance aspects, notably the choice of the order that minimizes the run time and memory requirements for a given flow configuration up to a desired accuracy will be examined on threedimensional applications in a future work.

An analysis of the aliasing phenomenon occurring for downstream propagation was also carried out, since its properties differ significantly when flow is present. It was shown that the aliasing resonances appear only when the upstream wave is under-resolved. Provided the grid is designed 
to ensure an accurate discretization of the shortest possible wavelength, the aliasing resonances were found to have a marginal impact in practice. It should be noted that even when the aliasing phenomenon is avoided it is still necessary to keep the interpolation error and the dispersion error (and hence the pollution effect) under control. This can be guided by the error estimator (40). 


\section{APPENDIX A: DISPERSION RELATION FOR THE QUADRANGULAR MESH}

The dispersion relation of the quadrangular mesh given in Figure 11 is obtained by projecting the exact solution in Equ. (41) on the quadrangle element. First consider the nodal degrees of freedom. At each node, the edge and bubble contributions vanish $\left(\tilde{p}_{n, m}^{e}=\tilde{p}_{n, m}^{b}=0\right)$ and we have

$$
\alpha_{n, m}^{v_{1}}=\alpha_{n, m}^{v_{2}} \mathrm{e}^{-\mathrm{i} k_{x} h_{x}}=\alpha_{n, m}^{v_{3}} \mathrm{e}^{-\mathrm{i}\left(k_{x} h_{x}+k_{y} h_{y}\right)}=\alpha_{n, m}^{v_{4}} \mathrm{e}^{-\mathrm{i} k_{y} h_{y}}=\mathrm{e}^{\mathrm{i} \mathbf{k} \cdot \mathbf{x}_{n, m}} .
$$

From the relation (48) it follows that each nodal degree of freedom on the grid $\alpha_{n, m}^{v_{1}}=\phi_{n, m}^{(1)}$ can be written under the form:

$$
\phi_{n, m}^{(1)}=\phi_{0}^{(1)} \tau_{x}^{n} \tau_{y}^{m},
$$

where $\phi_{0}^{(1)}$ does not depend on $(n, m)$ and we have introduced the following notations

$$
\tau_{x}=\mathrm{e}^{\mathrm{i} k_{x} h_{x}} \quad \text { and } \tau_{y}=\mathrm{e}^{\mathrm{i} k_{y} h_{y}} .
$$

The nodal unknowns form the first class of degrees of freedom. Similarly to the one dimensional case, the other classes are determined through an appropriate projection of the solution (41). On each of the four edges, the contribution of the bubble functions vanish $\left(\tilde{p}_{n, m}^{b}=0\right)$ and the trace of $\varphi_{q}^{e_{r}}(\xi, \eta)$ corresponds to a Lobatto function of order $q$. The $H_{0}^{1}\left(e_{r}\right)$ semi-norm [23] is used, which leads to minimizing the following residual:

$$
\int_{-1}^{1} \frac{\partial}{\partial \zeta}\left(p-\tilde{p}_{n, m}^{v}-\tilde{p}_{n, m}^{e}\right) \frac{\partial l_{q}}{\partial \zeta} \mathrm{d} \zeta=0, \quad q=2,3, \ldots, P,
$$

where $\zeta$ is a parametrization of the edge $e_{r}$. The orthogonality properties of the Lobatto functions are then used to compute each edge contribution:

$$
\begin{aligned}
& \alpha_{q, n, m}^{e_{1}}=\alpha_{q, n, m}^{e_{3}} \mathrm{e}^{-\mathrm{i} k_{y} h_{y}}=\mathrm{i} k_{x} \frac{h_{x}}{2} \int_{-1}^{1} \frac{\partial l_{q}}{\partial \xi} \mathrm{e}^{\mathrm{i} k_{x} h_{x}(\xi+1) / 2} \mathrm{~d} \xi \mathrm{e}^{\mathrm{i} \mathbf{k} \cdot \mathbf{x}_{n, m}}, \quad q=2,3, \ldots, P, \\
& \alpha_{q, n, m}^{e_{4}}=\alpha_{q, n, m}^{e_{2}} \mathrm{e}^{-\mathrm{i} k_{x} h_{x}}=\mathrm{i} k_{y} \frac{h_{y}}{2} \int_{-1}^{1} \frac{\partial l_{q}}{\partial \eta} \mathrm{e}^{\mathrm{i} k_{y} h_{y}(\eta+1) / 2} \mathrm{~d} \eta \mathrm{e}^{\mathrm{ik} \cdot \mathbf{x}_{n, m}}, \quad q=2,3, \ldots, P .
\end{aligned}
$$

Following (51), all edge degrees of freedom take the form

$$
\phi_{n, m}^{(g)}=\phi_{0}^{(g)} \tau_{x}^{n} \tau_{y}^{m},
$$

where the amplitude $\phi_{0}^{(g)}$ is independent of $(n, m)$. For a given order $q$, the degrees of freedom associated to the horizontal and vertical edges belong to two different classes. As a consequence, a total of $2(P-1)$ classes are needed for all edges unknowns on an element of order $P$. The contribution of internal degrees of freedom is then computed by projecting, in the sense of the $H^{1}\left(E^{Q}\right)$ norm, the residual $p-\tilde{p}_{n, m}^{v}-\tilde{p}_{n, m}^{e}$ onto the space formed by the bubble functions. One finally obtains:

$$
\alpha_{q_{1}, q_{2}, n, m}^{b}=-k_{x} k_{y} \frac{h_{x} h_{y}}{4} \int_{-1}^{1} \frac{\partial l_{q_{1}}}{\partial \xi} \mathrm{e}^{\mathrm{i} k_{x} h_{x}(\xi+1) / 2} \mathrm{~d} \xi \int_{-1}^{1} \frac{\partial l_{q_{2}}}{\partial \eta} \mathrm{e}^{\mathrm{i} k_{y} h_{y}(\eta+1) / 2} \mathrm{~d} \eta \mathrm{e}^{\mathrm{i} \mathbf{k} \cdot \mathbf{x}_{n, m}} .
$$

This completes the proof that all system unknowns are of the form (52). On the $E_{n, m}$ element, the shape functions related to node 1 , edges 1 and 4 and all internal shape functions form the 'minimum' mesh stencil. Through successive translations of $h_{x}$ and $h_{y}$ in the two cartesian directions of this stencil, all the approximation basis of the model can be constructed. This leads, for a periodic quadrangular mesh of element of order $P$, to a total of $N=P^{2}$ classes, which represent as many independent equations in the algebraic system. Similarly to the one-dimensional case, for all classes of degrees of freedom $g$, the corresponding equation reads

$$
\sum_{r=1}^{N} \sum_{n, m} A_{n, m}^{(g, r)} \phi_{n, m}^{(r)}=0, \quad g=1,2, \ldots, N,
$$


where $A_{n, m}^{(g, r)}$ denotes the contribution of $\phi_{n, m}^{(r)}$ in the equation for the degrees of freedom of class $g$. By substituting (52) in equations (54), the dispersion relation of the discrete model is also of the form (28) with R a matrix of size $P^{2}$ and $\phi_{0}$ the interpolation vector containing the contributions of the different classes of shape functions. 


\section{APPENDIX B: DISPERSION RELATION FOR THE TRIANGULAR MESH}

For the triangle meshes, we follow the same approach as in Appendix A for the quadrangle mesh. The different classes of degrees of freedom are first determined through interpolation and projection of the plane wave solution (41). After some algebra, the following expressions are obtained for the nodal and edge contributions of the stencil A:

$$
\begin{aligned}
& \alpha_{n, m}^{v_{1}}=\alpha_{n, m}^{n_{2}} \mathrm{e}^{-\mathrm{i} k_{x} h_{x}}=\alpha_{n, m}^{n_{3}} \mathrm{e}^{-\mathrm{i} k_{y} h_{y}}=\mathrm{e}^{\mathrm{i} \mathbf{k} \cdot \mathbf{x}_{n, m}}, \\
& \alpha_{q, n, m}^{e_{1}}=\mathrm{i} k_{x} \frac{h_{x}}{2} \int_{-1}^{1} \frac{\partial l_{q}(\xi)}{\partial \xi} \mathrm{e}^{\mathrm{i} k_{x} h_{x}(\xi+1) / 2} \mathrm{~d} \xi \mathrm{e}^{\mathrm{i} \mathbf{k} \cdot \mathbf{x}_{n, m}}, \quad \forall q \geq 2, \\
& \alpha_{q, n, m}^{e_{2}}=\mathrm{i} \frac{\left(k_{x} h_{x}-k_{y} h_{y}\right)}{2}(-1)^{q} \int_{-1}^{1} \frac{\partial l_{q}(\xi)}{\partial \xi} \mathrm{e}^{\mathrm{i}\left(k_{x} h_{x}(\xi+1) / 2+k_{y} h_{y}(1-\xi) / 2\right)} \mathrm{d} \xi \mathrm{e}^{\mathrm{i} \mathbf{k} \cdot \mathbf{x}_{n, m}}, \quad \forall q \geq 2, \\
& \alpha_{q, n, m}^{e_{3}}=\mathrm{i} k_{y} \frac{h_{y}}{2} \int_{-1}^{1} \frac{\partial l_{q}(\eta)}{\partial \eta} \mathrm{e}^{\mathrm{i} k_{y} h_{y}(\eta+1) / 2} \mathrm{~d} \eta \mathrm{e}^{\mathrm{i} \mathbf{k} \cdot \mathbf{x}_{n, m}}, \quad \forall q \geq 2 .
\end{aligned}
$$

It follows that all the nodal degrees of freedom belong to the same class. However, at a given order $q$, three distinct classes of edge degrees of freedom are found, corresponding respectively to the vertical, horizontal and diagonal edges. These expressions indicate that all edge degrees of freedom can be written in the form:

$$
\phi_{n, m}^{(g)}=\phi_{0}^{(g)} \tau_{x}^{n} \tau_{y}^{m} .
$$

The same conclusion holds for the stencil B, which theoretical projections are given below

$$
\begin{aligned}
& \alpha_{n, m}^{v_{1}}=\alpha_{n, m}^{v_{2}} \mathrm{e}^{-\mathrm{i} k_{x} h_{x}}=\alpha_{n, m}^{v_{3}} \mathrm{e}^{-\mathrm{i}\left(k_{y} h_{y}+k_{x} \frac{h_{x}}{2}\right)}=\mathrm{e}^{\mathrm{i} \mathbf{k} \cdot \mathbf{x}_{n, m}}, \\
& \alpha_{k, n, m}^{e_{1}}=\mathrm{i} k_{x} \frac{h_{x}}{2} \int_{-1}^{1} \frac{\partial l_{k}(\xi)}{\partial \xi} \mathrm{e}^{\mathrm{i} k_{x} h_{x}(\xi+1) / 2} \mathrm{~d} \xi \mathrm{e}^{\mathrm{i} \mathbf{k} \cdot \mathbf{x}_{n, m}}, \quad \forall k \geq 2, \\
& \alpha_{k, n, m}^{e_{2}}=\mathrm{i}\left(k_{x} \frac{h_{x}}{4}-k_{y} \frac{h_{y}}{2}\right)(-1)^{k} \int_{-1}^{1} \frac{\partial l_{k}(\xi)}{\partial \xi} \mathrm{e}^{\mathrm{i}\left(k_{x} h_{x}(\xi+3) / 4+k_{y} h_{y}(1-\xi) / 2\right)} \mathrm{d} \xi \mathrm{e}^{\mathrm{i} \mathbf{k} \cdot \mathbf{x}_{n, m}}, \quad \forall k \geq 2, \\
& \alpha_{k, n, m}^{e_{3}}=\mathrm{i}\left(k_{x} \frac{h_{x}}{4}+k_{y} \frac{h_{y}}{2}\right) \int_{-1}^{1} \frac{\partial l_{k}(\eta)}{\partial \eta} \mathrm{e}^{\mathrm{i}\left(k_{x} h_{x}(\eta+1) / 4+k_{y} h_{y}(\eta+1) / 2\right)} \mathrm{d} \eta \mathrm{e}^{\mathrm{i} \mathbf{k} \cdot \mathbf{x}_{n, m}}, \quad \forall k \geq 2,
\end{aligned}
$$

except that all classes of degrees of freedom now take the following form

$$
\phi_{n, m}^{(g)}=\phi_{0}^{(g)} \tau_{x}^{n} \tau_{x y}^{m},
$$

where $\tau_{x y}=\mathrm{e}^{\mathrm{i} k_{x} h_{x} / 2+\mathrm{i} k_{y} h_{y}}$. Note that the projection on the $E_{n, m}^{I I}$ element is not necessary since all nodal and edge degrees of freedom have already been associated to a class.

Concerning the internal unknowns contribution, the residual $p-\tilde{p}_{n, m}^{v}-\tilde{p}_{n, m}^{e}$ of element $E_{n, m}^{I}$ is projected over the space spanned by its bubble functions in the $H^{1}\left(E^{T}\right)$ semi-norm [23]. The minimization problem then leads to solving the following equation:

$$
\int_{E^{T}} \nabla\left(p-\tilde{p}_{n, m}^{v}-\tilde{p}_{n, m}^{e}-\tilde{p}_{n, m}^{b}\right) \cdot \nabla\left(\varphi_{r_{1}, r_{2}}^{b}\right) \mathrm{d} E^{T}=0,
$$

which can also be written:

$$
\begin{aligned}
\sum_{q_{1}=1}^{P} \sum_{q_{2}=1}^{\left(P-1-q_{1}\right)} \alpha_{q_{1}, q_{2}, n, m}^{b} \int_{E^{T}} \nabla\left(\varphi_{q_{1}, q_{2}}^{b}\right) & \cdot \nabla\left(\varphi_{r_{1}, r_{2}}^{b}\right) \mathrm{d} E^{T} \\
& =\int_{E^{T}} \nabla\left(p-\tilde{p}_{n, m}^{v}-\tilde{p}_{n, m}^{e}\right) \cdot \nabla\left(\varphi_{q_{1}, q_{2}}^{b}\right) \mathrm{d} E^{T} .
\end{aligned}
$$

The former equation represents a linear algebraic system of size $(P-2)(P-1) / 2$ for the bubble unknowns $\alpha_{q_{1}, q_{2}, n, m}^{b}$ of element $E_{n, m}^{I}$. Let us denote $\boldsymbol{\alpha}^{b, I}$, the vector containing these bubble 
degrees of freedom. Using relations (55) and (57), the right-hand side can be factorized and (60) becomes:

$$
\mathrm{B} \alpha^{b, I}=\mathrm{S}^{I} \tau_{x}^{n} \tau_{y}^{m} \quad \text { and } \quad \mathrm{B} \alpha^{b, I}=\mathrm{S}^{I} \tau_{x}^{n} \tau_{x y}^{m},
$$

respectively for the triangular meshes $\mathrm{A}$ and $\mathrm{B}$, where $\mathrm{S}^{I}$ does not depend on $(n, m)$. The same operation applied now on element $E_{n, m}^{I I}$ leads to a similar expression:

$$
\mathrm{B} \alpha^{b, I I}=\mathrm{S}^{I I} \tau_{x}^{n} \tau_{y}^{m} \quad \text { and } \quad \mathrm{B} \alpha^{b, I I}=\mathrm{S}^{I I} \tau_{x}^{n} \tau_{x y}^{m},
$$

respectively for the triangle mesh $\mathrm{A}$ and the triangle mesh $\mathrm{B}$, where $\mathrm{S}^{I I}$ does not depend on $(n, m)$. It can be easily shown that $\mathrm{S}^{I}$ and $\mathrm{S}^{I I}$ are independent, thus the bubble degrees of freedom of elements $E_{n, m}^{I}$ and $E_{n, m}^{I I}$ form two distinct classes at a given order. We have proven that all unknowns can write under the form (56) or (58) respectively for the mesh A and mesh B. A total of $N=1+3 \times(P-1)+2 \times(P-1)(P-2) / 2$, so simply $N=P^{2}$ different classes were identified. Following the methodology described for the quadrangular mesh, the dispersion relation of the numerical model can be readily obtained. 


\section{REFERENCES}

1. Babuška I, Sauter S. Is the pollution effect of the FEM avoidable for the Helmholtz equation considering high wavenumbers? SIAM Journal of Numerical Analysis 1997; 34:2392-2423.

2. Melenk J, Babuška I. The partition of unity element method: basic theory and applications. Computers Methods in Applied Mechanics and Engineering 1996; 139:289-314.

3. Farhat C, Harari I, Franca L. The discontinuous enrichment method. Computers Methods in Applied Mechanics and Engineering 2001; 190:6455-6479.

4. Cessenat O, Després B. Application of an ultra-weak variational formulation of elliptic PDEs to the twodimensional Helmholtz problem. SIAM Journal of Numerical Analysis 1998; 35:255-299.

5. Astley R, Gamallo P. Special short wave elements for flow acoustics. Computer Methods in Applied Mechanics and Engineering 2005; 194:341-353.

6. Gamallo P, Astley R. The partition of unity finite element method for short wave acoustic propagation on nonuniform potential flows. International Journal for Numerical Methods in Engineering 2006; 65:425-444.

7. Gabard G. Discontinuous Galerkin methods with plane waves for time-harmonic problems. Journal of Computational Physics 2007; 225:1961-1984.

8. Sevilla R, Fernández-Méndez S, Huerta A. 3D NURBS-enhanced finite element method (NEFEM). Internat. J. Numer. Methods Engrg. 2011; 88(2):103-125.

9. Brigham J, Aquino W, Aguilo M, Diamessis P. A spectral finite element approach to modeling soft solids excited with high frequency harmonic loads. Computer Methods in Applied Mechanical Engineering 2011; 200:692-698.

10. Thompson L, Pinsky M. Complex wave number Fourier analysis of the $p$-version finite element method. Computational Mechanics 1994; 13:255-275.

11. Mulder W. Spurious modes in finite-element discretizations of the wave equation may not be all that bad. Applied Numerical Mathematics 1999; 30:425-445.

12. Ihlenburg F, Babuška I. Finite element solution to the Helmholtz equation with high wavenumber. Part I: the $h$ version of the FEM. Computational Methods in Applied Mechanical Engineering 1995; 30:9-37.

13. Ihlenburg F, Babuška I. Finite element solution to the Helmholtz equation with high wavenumber. Part II: the $h p$ version of the FEM. SIAM Journal of Numerical analysis 1997; 34:315-358.

14. Deraemaeker A, Babuška I, Bouillard P. Dispersion and pollution of the FEM solution for the Helmholtz equation in one, two and three dimensions. International Journal for Numerical Methods in Engineering 1999; 46:471-499.

15. Ainsworth M. Discrete dispersion relation for hp-version finite element approximation at high wave number. SIAM Journal of Numerical Analysis 2004; 42(2):553-575.

16. Cottrell J, Reali A, Bazilevs Y, Hughes T. Isogeometric analysis of structural vibrations. Computer Methods in Applied Mechanics and Engineering 2006; 195:5257-5296.

17. Brillouin L. Wave propagation in periodic structures. McGraw-Hill, 1946.

18. Petersen S, Dreyer D, von Estorff O. Assessment of finite and spectral element shape functions for efficient iterative simulations of interior acoustics. Computers Methods in Applied Mechanics and Engineering 2006; 195:64636478.

19. Vos P, Sherwin S, Kirby R. From $h$ to $p$ efficiently: Implementing finite and spectral $/ h p$ element discretisations to achieve optimal performance at low and high order approximations. Journal of Computational Physics 2010; 229:5161-5181.

20. Gabard G, Astley R, Ben Tahar M. Stability and accuracy of finite element methods for flow acoustics. I: General theory and application to one-dimensional propagation. International Journal for Numerical Methods in Engineering 2005; 63:947-973.

21. Gabard G, Astley R, Ben Tahar M. Stability and accuracy of finite element methods for flow acoustics. II: Twodimensional effects. International Journal for Numerical Methods in Engineering 2005; 63:947-973.

22. Astley R. Numerical methods for noise propagation in moving flows, with application to turbofan engines. Acoustical Science and Technology 2009; 30(4):227-239.

23. Šolín P, Segeth K, Doležel I. Higher-order finite element methods. Chapman \& Hall: New York, 2003.

24. Szabó B, Babuška I. Finite element analysis. Wiley, 1991.

25. Dunavant D. High degree efficient symmetrical Gaussian quadrature rules for the triangle. International Journal for Numerical Methods in Engineering 1985; 21:1129-1148.

26. Ihlenburg F, Babuška I. Dispersion analysis and error estimation of Galerkin finite element methods for the Helmholtz equation. International journal for numerical methods in engineering 1995; 38:3745-3774.

27. Harari I, Nogueira C. Reducing dispersion of linear triangular elements for the Helmholtz equation. Journal of Engineering Mechanics 2002; 128:351-358. 